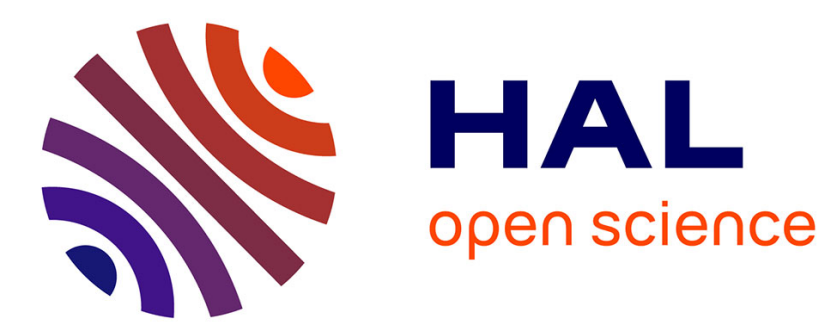

\title{
An axiomatic version of Zariski's patching theorem
} Olivier Piltant

\section{To cite this version:}

Olivier Piltant. An axiomatic version of Zariski's patching theorem. 2010. hal-00612635

\section{HAL Id: hal-00612635 \\ https://hal.science/hal-00612635}

Preprint submitted on 29 Jul 2011

HAL is a multi-disciplinary open access archive for the deposit and dissemination of scientific research documents, whether they are published or not. The documents may come from teaching and research institutions in France or abroad, or from public or private research centers.
L'archive ouverte pluridisciplinaire HAL, est destinée au dépôt et à la diffusion de documents scientifiques de niveau recherche, publiés ou non, émanant des établissements d'enseignement et de recherche français ou étrangers, des laboratoires publics ou privés. 


\title{
AN AXIOMATIC VERSION OF ZARISKI'S PATCHING THEOREM
}

\author{
OLIVIER PILTANT
}

\begin{abstract}
We state six axioms concerning any regularity property $P$ in a given birational equivalence class of algebraic threefolds. Axiom 5 states the existence of a local uniformization in the sense of valuations for $P$. If axioms 1 to 4 are satisfied by $P$, then the function field has a projective model which is everywhere regular w.r.t. $P$. Axiom 6 ensures the existence of $P$-resolution of singularities for any projective model. Applications concern resolution of singularities of vector fields and a weak version of Hironaka's Strong Factorization Conjecture for birational morphisms of nonsingular projective threefolds, both of them in characteristic zero.
\end{abstract}

\section{InTRODUCTION.}

Zariski's Patching Theorem ([33], Fundamental Theorem on p. 539) was one of his main achievements in the field of resolution of singularities. The content of this theorem is that local uniformization of valuations in a three-dimensional function field $K$ over a ground field $k$ of characteristic zero implies the existence of a nonsingular projective model of $K$. Since Zariski had proved the existence of a local uniformization for any valuation and any function field over a ground field of characteristic zero in [31], existence of nonsingular projective models was established for threefolds.

Hironaka's Resolution of Singularities [18] in 1964 closed this approach in characteristic zero. Indeed, Hironaka's theorem proves much more than the existence of nonsingular projective models as considered by Zariski. Moreover, since de Jong's work on alterations [22] some fifteen years ago, a much simpler proof of the existence of nonsingular projective models in characteristic zero and all dimensions is now available [4] [6]. On the other hand, the Patching Theorem has never been extended to dimensions higher than three (without assuming beforehand the existence of nonsingular projective models).

In positive characteristics, the situation is very different. The best result up to date is proved in a joint work of the author with V. Cossart (conjecture 3.1 in [12], stated in the introduction and proved in [13]) for threefolds over any ground field $k$ of characteristic $p>0$ which is differentially finite over a perfect field. This proof actually uses an extension of Zariski's Patching Theorem (proposition 4.8 in [12]) to arbitrary ground fields of positive characteristics.

Since Zariski's time, several resolution type problems have been considered. Resolution of Singularities of vector fields is considered by F. Cano in [7], and that of

CNRS and LMV, UMR 8100, University of Versailles. Partially supported by "Ayudas a la Movilidad de Profesores" of the University of Valladolid. 
foliations in [8] [9]. Hironaka's Strong Factorization Conjecture for birational morphisms by blowing ups and blowing down regular centers is also a resolution type problem (stated in [18], a weaker form is proved in [5] [29]). All of these problems are open in dimension four or more. In dimension three and characteristic zero, the question for foliations has been settled by F. Cano in [9].

A local formulation of each of these problems can be given in terms of valuation theory, which is called Local Uniformization (axiom 5 in section 2). Specifying a valuation $\operatorname{ring} V$ in a function field $K$, with $k \subset V$ and $Q F(V)=K$, is equivalent to specifying a center $x_{V}$ on each projective model $X / k$ of $K$, compatible with morphisms. The Local Uniformization problem is in general substantially simpler than the Resolution problem because the concrete resolution strategy is allowed to be adapted to the valuation. Also valuation rings are algebraic objects with a simple combinatorial structure in which terms resolution problems can be tracked more easily.

The main applications of this article concern vector fields and a weak version of Hironaka's Strong Factorization Conjecture in dimension three [18]. Our main result (theorem 2.5 below) reduces (global) Resolution to Local Uniformisation in each of these cases. Combining it with their Local Uniformization theorem, F. Cano, C. Roche and M. Spivakovsky ([10] theorem 2) obtain Resolution for vector fields (restated as corollary 4.9).

Combining theorem 2.5 with K. Karu's Local Uniformization for the Hironaka Strong Factorisation Conjecture, thorem 0.1 [23], we obtain our globalized version in theorem 4.10. We emphasize that our result builds up projective birational morphisms which are locally a composition of blowing ups along regular centers, a fairly wider class than that of compositions of blowing ups with regular centers. On the other hand, germs of such morphisms have been used quite systematically to build up local uniformizations: Zariski's Perron transforms [31], Cutkosky's MTSs [14], Karu's star subdivisions in the toric case [23] and F. Cano, C. Roche and M. Spivakovsky's Puiseux packages are examples. From this viewpoint, one can understand the assumptions (axioms 1 to 4 and 6) of theorem 2.5 as a criterion for arbitrary regularity properties in dimension three in order that the only obstruction to Resolution be Local Uniformization.

Our setup consists in studying any abstract regularity property (definition 2.1) in a given function field $K$ of dimension three over any ground field $k$. In section 2 , we introduce six axioms to be verified by $P: 1$. openness, 2 . stability by normalization and normalized blowing ups of points, 3. making curves "permissible" at regular points, 4. principalization of ideals, 5. local uniformization and 6. making curves "permissible" at singular points. The first five axioms ensure the existence of a model of $K$ which is everywhere regular w.r.t. $P$ (theorem 2.4). Adding axiom 6 ensures Resolution of Singularities in the birational equivalence class of $K$ (theorem 2.5).

Section 3 contains those examples of regularity properties we are interested in: reducing multiplicity (property $P_{\mu}$, example 3.4 ), getting a vector field to be logelementary (property $P_{\delta}$, example 3.6), and solving our local version of Hironaka's Strong Factorization Conjecture (property $P_{R S(Y)}$, definitions 3.8 and 3.9). All of this is developed in a relative context, that is, to any given regularity property $P$, we associate a new one refining $P$ by requiring furthermore regularity w.r.t. one of the above problems. Our axioms 1, 2, 3 and 6 also have good behaviour by taking the boolean operation $P_{1} \wedge P_{2}$ of two regularities properties $P_{1}$ and $P_{2}$ (proposition 3.11). 
Section 4 contains the main applications: checking that certain regularity properties of $P_{\delta}$-type and $P_{R S\left(Y_{1}\right)} \wedge P_{R S\left(Y_{2}\right)}$-type verify axioms 1 to 6 , we deduce our main theorems (proposition 4.8 and theorem 4.10). The main difficulty consists in proving principalization of ideals for $P_{\delta}$ (axiom 4, proposition 4.2) and making curves "permissible" at singular points for $P_{R S(Y)}$ (axiom 6, proposition 4.7).

Finally, sections 5 and 6 consist in adapting Zariski's proof of the Patching Theorem to any regularity property $P$ satisfying our axioms. We conclude by suggesting some open problems, the most challenging of which is problem 7.1: to get a constructive version of theorem 2.5 .

\section{P-Regularity and statement of main Result.}

All along this article, we consider a field $k$ of arbitrary characteristic $p \geq 0$ and a function field $K$ of transcendence degree three over $k$.

Definition 2.1. A regularity property $P$ in $K$ is an application from the set of all local rings $R \subseteq K$ which are localization at a prime ideal of an integral $k$-algebra of finite type such that $Q F(R)=K$ to the set \{regular, singular\}. Those $R$ mapping to \{regular\} (resp. \{singular\}) are then called P-regular (resp. P-singular). Given any proper model $X / k$ of $K$, we denote

$$
\operatorname{Reg} \mathrm{P}(X):=\left\{x \in X \mid \mathcal{O}_{X, x} \text { is } P-\operatorname{regular}\right\}, \operatorname{Sing} \mathrm{P}(X):=X \backslash \operatorname{Reg} \mathrm{P}(X) .
$$

Remark: although our applications concern regularity properties $P$ which are refinements of the usual regularity property (i.e. $\operatorname{RegP}(X) \subseteq X_{\text {reg }}$ ) for any projective model $X / k$ of $K$ ), we do not make this extra assumption unless otherwise stated (example 3.6 and section 4 ).

We now introduce various axioms to be satisfied by $P$.

Axiom 1 (Openness). For any projective model $X / k$ of $K$, the set $\operatorname{RegP}(X)$ is a nonempty open set of $X$ for its Zariski topology.

We are interested in defining a class of projective birational morphisms $\pi: X^{\prime} \rightarrow X$ such that $\pi^{-1}(\operatorname{RegP}(X)) \subseteq \operatorname{RegP}\left(X^{\prime}\right)$. A first step is the following axiom:

Axiom 2 (Stability by normalization and normalized blowing up). The following holds for any projective model $X / k$ of $K$ :

(i) let $n: X_{\text {norm }} \rightarrow X$ be the normalization of $X$. Then $n^{-1}(\operatorname{RegP}(X)) \subseteq$ $\operatorname{Reg} \mathrm{P}\left(X_{\text {norm }}\right)$.

(ii) let $x \in \operatorname{RegP}(X)$ be such that $\mathcal{O}_{X, x}$ is normal, $Z:=\overline{\{x\}}$ and $\pi: \tilde{X} \rightarrow X$ be the normalized blowing up of $X$ along $Z$. There exists a nonempty open set $U_{Z} \subset Z$ such that $\pi^{-1}(U) \subseteq \operatorname{RegP}(\tilde{X})$.

If $\operatorname{dim} \mathcal{O}_{X, x}=1, \pi$ is an isomorphism above $x$, so (ii) is always satisfied in this case. If $x$ is a closed point, i.e. $\operatorname{dim} \mathcal{O}_{X, x}=3$, and (ii) of axiom 2 holds, we have $U_{Z}=Z$ necessarily. In case $\operatorname{dim} \mathcal{O}_{X, x}=2$, we need some control on the open set $U_{Z}$. This leads to the following definitions.

Definition 2.2. Assume that the regularity property $P$ verifies axioms 1 and 2 and keep notations and assumptions as in the statement of (ii) of axiom 2. 
We say that $Z$ is $P$-permissible at some point $z \in Z$ if $z \in \operatorname{RegP}(X)$, $z$ is a regular point of $Z$ in the usual sense, $\mathcal{O}_{X, z}$ is normal, and $U_{Z}$ can be chosen in such a way that $z \in U_{Z}$.

We say that $Z$ is stably $P$-permissible at some point $z \in Z$ if for any finite sequence

$$
X=: X_{0} \leftarrow X_{1} \leftarrow \cdots \leftarrow X_{n}
$$

of normalized blowing ups at points $z_{i-1} \in X_{i-1}$ such that $z_{0}:=z$ and for $1 \leq i \leq n$, $z_{i}$ maps to $z_{i-1}$ and belongs to the strict transform $Z_{i}$ of $Z$ in $X_{i}, Z_{n}$ is $P$-permissible at $z_{n}$.

Note that $Z$ is stably $P$-permissible at its generic point $x$ by definition, since $Z_{1}=\emptyset$ in (1) when $z=x$. In particular, any closed point in any normal model of $K$ is stably $P$-permissible if $P$ verifies axioms 1 and 2 .

Example 2.3. Take $P_{\text {reg }}$ to be the usual regularity property, i.e. $\mathcal{O}_{X, x}$ has property $P_{\text {reg }}$ iff $\mathcal{O}_{X, x}$ is a regular local ring. To avoid confusions, we will denote the corresponding regularity open sets $\operatorname{Reg}_{\mathrm{reg}}(X)$ by $X_{\mathrm{reg}}$ for any projective model $X / k$ of $K$.

It is immediately seen that $P_{\text {reg }}$ verifies axioms 1 and 2. If $X / k$ is a projective model of $K, Z \subset X$ an integral curve and $z \in X_{\mathrm{reg}}$ is a regular point of $Z$, then $Z$ is stably $P$-permissible at $z$.

Axiom 3 (Permissibilizing curves). The following holds for any normal projective model $X / k$ of $K$ and for any $x \in \operatorname{RegP}(X)$ such that $\operatorname{dim} \mathcal{O}_{X, x}=2$ : there exists a finite sequence

$$
X=: X_{0} \leftarrow X_{1} \leftarrow \cdots \leftarrow X_{n}=: X^{\prime}
$$

of normalized blowing ups at closed points such that the strict transform $Z^{\prime}$ of $Z=\overline{\{x\}}$ in $X^{\prime}$ is stably $P$-permissible at all points $z^{\prime} \in Z^{\prime} \cap \operatorname{RegP}\left(X^{\prime}\right)$.

It is worth noting that, if $P$ verifies axiom 2 , we necessarily have $\pi^{-1}(\operatorname{RegP}(X)) \subseteq$ $\operatorname{RegP}\left(X^{\prime}\right)$, where $\pi: X^{\prime} \rightarrow X$ is the composed map in (2). In particular, any integral curve in a normal variety whose generic point is $P$-regular is stably $P$-permissible at all but finitely many closed points if $P$ verifies axioms 1,2 and 3 .

Note that one gets equivalent notions for axioms 1, 2 and 3 if the word "projective" is replaced with "proper", since the question is local on $X$.

Axiom 4 (Principalization). For any normal projective model $X / k$ of $K$ and any ideal sheaf $\mathcal{I} \subset \mathcal{O}_{X}$, there exists a projective birational morphism $\pi: \tilde{X} \rightarrow X$ having the following properties:

(i) $\mathcal{I O}_{\pi^{-1}(\operatorname{RegP}(X))}$ is locally principal.

(ii) $\pi^{-1}(\operatorname{RegP}(X)) \subseteq \operatorname{RegP}(\tilde{X})$.

(iii) the induced map $\pi^{-1}(\operatorname{RegP}(X) \cap U) \rightarrow \operatorname{RegP}(X) \cap U$ is an isomorphism, where $U \subseteq X$ is the open set

$$
U:=\left\{x \in X \mid \mathcal{I}_{x} \text { is principal }\right\} .
$$

Note that we do not require $\pi$ to be a composition of elementary blowing ups (for example at integral or at regular centers) in this definition. We obtain an equivalent 
formulation of axiom 4 by replacing (i) by the stronger property:

(i) $\mathcal{I O}_{\tilde{X}}$ is locally principal.

Namely, since $\mathcal{I} \mathcal{O}_{\pi^{-1}(\operatorname{RegP}(X))}$ is already locally principal, blowing up $\mathcal{I O}_{\tilde{X}}$ achieves (i)' without affecting neither (ii) nor (iii).

Finally, note that $X \backslash U$ has dimension at most one, since $X$ is assumed to be normal in the definition of axiom 4 , and that $\pi$ is the blowing up along an ideal sheaf $\mathcal{J} \subseteq \mathcal{O}_{X}$ such that

(iii)' $\mathcal{J} \mathcal{O}_{\operatorname{RegP}(X) \cap U}$ is locally principal.

We now introduce the local uniformization axiom. Let $V$ be a $k$-valuation ring of $K$, i.e. $k \subset V$ and $Q F(V)=K$. For any proper model $X / k$ of $K, V$ has a unique center $x_{V} \in X$. There is a one-to-one correspondence

$$
\{k \text { - valuation rings of } K\} \leftrightarrow \varliminf \lim X, V \mapsto\left(x_{V}\right)_{X},
$$

where the inverse limit is taken over all proper models $X / k$ of $K$. By Chow's lemma, we get an equivalent notion if the word "proper" is replaced with "projective". The following axiom is a weak statement of Local Uniformization for property $P$. Similarly, we get an equivalent notion if the word "proper" is replaced with "projective", since the question is local on $X$.

Axiom 5 (Local Uniformization). For each $k$-valuation ring $V / k$ of $K$, there exists a proper model $X / k$ of $K$ such that the center $x_{V} \in X$ of $V$ satisfies $x_{V} \in \operatorname{RegP}(X)$.

Our axiomatic version of Zariski's patching theorem goes as follows:

Theorem 2.4. Let $P$ be a regularity property verifying axioms 1 to 5 and $X / k$ be a projective model of $K$. There exists a birational projective morphism $\pi: X^{\prime} \rightarrow X$, $X^{\prime}$ normal, such that $\operatorname{RegP}\left(X^{\prime}\right)=X^{\prime}$.

This result can be strengthened by introducing a new axiom:

Axiom 6. The following holds for any normal projective model $X / k$ of $K$ and for any $x \in \operatorname{RegP}(X)$ such that $\operatorname{dim} \mathcal{O}_{X, x}=2$ : there exists a finite sequence

$$
X=: X_{0} \leftarrow X_{1} \leftarrow \cdots \leftarrow X_{n}=: X^{\prime}
$$

of normalized blowing ups at closed points such that the strict transform $Z^{\prime}$ of $Z:=\overline{\{x\}}$ in $X^{\prime}$ verifies $Z^{\prime} \subset \operatorname{RegP}\left(X^{\prime}\right)$.

Theorem 2.5. Let $P$ be a regularity property verifying axioms 1 to 6 and $X / k$ be a normal projective model of $K$. There exists a birational projective morphism $\pi$ : $X^{\prime} \rightarrow X, X^{\prime}$ normal, such that $\operatorname{RegP}\left(X^{\prime}\right)=X^{\prime}$ and the induced map

$$
\pi^{-1}(\operatorname{RegP}(X)) \rightarrow \operatorname{RegP}(X)
$$

is an isomorphism.

Remark: if a regularity property $P$ verifies some of axioms $1,2,3$ or 6 , so does $P_{\text {norm }}$, where for any projective model $X / k$ of $K$,

$$
\operatorname{RegP}_{\text {norm }}(X):=\operatorname{RegP}(X) \cap\left\{x \in X \mid \mathcal{O}_{X, x} \text { is normal }\right\} .
$$


The same fact holds for axioms 4 and 5 provided $P$ verifies (i) of axiom 2. Finally, $X^{\prime}$ satisfies the conclusion of theorem 2.4 (resp. of theorem 2.5) w.r.t. $P_{\text {norm }}$ whenever $X^{\prime}$ satisfies the conclusion of theorem 2.4 (resp. of theorem 2.5) w.r.t. $P$.

\section{BAsic Properties of $P$-REgularity AND APPlications.}

In this section, we point out some simple consequences of axioms 1 to 6 , then introduce several examples of regularity properties which fit into our framework.

Proposition 3.1. Let $P$ be any regularity property verifying axioms 1, 2 and 5 . Let $X / k$ be a normal and projective model of $K$. Then

$$
\operatorname{dim}(\operatorname{SingP}(X)) \leq 1 .
$$

Proof. Since $\operatorname{RegP}(X)$ is Zariski open by axiom 1 , it is sufficient to prove that $x \in$ $\operatorname{RegP}(X)$ whenever $\operatorname{dim} \mathcal{O}_{X, x}=1$. Then $V:=\mathcal{O}_{X, x}$ is a prime divisor.

By axiom 5, there exists a projective model $Y / k$ of $K$ such that the center $y_{V} \in Y$ of $V$ belongs to $\operatorname{RegP}(Y)$. Let

$$
Y=: Y_{0} \leftarrow Y_{1} \leftarrow \cdots \leftarrow Y_{n} \leftarrow \cdots
$$

be the sequence of normalized blowing ups defined as follows: for $i \geq 1, Y_{i}$ is the normalized blowing up along $\overline{\left\{y_{i-1, V}\right\}}$, where $y_{i-1, V}$ is the center of $V$ in $Y_{i-1}$. Using a clasical argument of Zariski ([30] first paragraph in the proof of theorem 10 which works in all dimensions), the sequence of local rings $\mathcal{O}_{Y_{i}, y_{i, V}}$ gets constant and equal to $V=\mathcal{O}_{X, x}$ for $i \geq i_{0}$. On the other hand, $y_{i, V} \in \operatorname{RegP}\left(Y_{i}\right)$ for all $i \geq 0$ by (i) and (ii) of axiom 2 and the conclusion follows.

Proposition 3.2. Let $P$ be any regularity property verifying axioms 1, 2 and 3 (resp. 1, 2, 3 and 6) and $Y / k$ be a fixed projective model of $K$. We define a regularity property $P_{Y}$ as follows: for any projective model $X / k$ of $K$,

$$
\operatorname{RegP}_{Y}(X):=\operatorname{RegP}(X) \cap\{x \in X \mid X \cdots \rightarrow Y \text { is defined }\} .
$$

Then $P_{Y}$ verifies axioms 1, 2 and 3 (resp. 1, 2, 3 and 6). If moreover $P$ verifies axiom 4 (resp. 4 and 5), then $P_{Y}$ also verifies axiom 4 (resp. 4 and 5).

Proof. Let $X / k$ be a projective model of $K$ and $Y^{\prime}$ be the closure of the graph of the rational map $X \cdots \rightarrow Y$, so $Y^{\prime}$ dominates both of $X$ and $Y$. Then $Y^{\prime} \rightarrow X$ is projective, hence the blowing up along a certain ideal sheaf $\mathcal{I} \subseteq \mathcal{O}_{X}$. This gives the following description:

$$
\operatorname{RegP}_{Y}(X):=\operatorname{RegP}(X) \cap\left\{x \in X \mid \mathcal{I}_{x} \text { is locally principal }\right\} .
$$

Hence $P_{Y}$ verifies axioms 1,2 and 3 , since $P$ does.

We now use axiom 4 for $P$ to the pair $(X, \mathcal{I})$. This produces a projective birational morphism $\pi: \tilde{X} \rightarrow X$ with the following properties:

(i') $\operatorname{RegP}_{Y}(\tilde{X})=\operatorname{RegP}(\tilde{X})$.

(ii) $\pi^{-1}(\operatorname{RegP}(X)) \subseteq \operatorname{RegP}_{Y}(\tilde{X})$.

(iii) the induced map $\pi^{-1}\left(\operatorname{RegP}_{Y}(X)\right) \rightarrow \operatorname{RegP}_{Y}(X)$ is an isomorphism.

In other terms, we may substitute $X$ by $\tilde{X}$ in order to prove that $P_{Y}$ verifies axiom 4. Since $\operatorname{RegP}_{Y}\left(X^{\prime}\right)=\operatorname{RegP}\left(X^{\prime}\right)$ for every projective model $X^{\prime} / k$ of $K$ birationally 
dominating $\tilde{X}$, axiom 4 for $P_{Y}$ is a direct consequence of axiom 4 for $P$.

Assume that $P$ verifies axiom 6 . Let $X / k$ be a normal projective model of $K$ and $Z \subset X$ be an integral curve such that $Z \cap \operatorname{RegP}_{Y}(X) \neq 0$. Then $\mathcal{I O}_{X, Z}$ is locally principal. To prove that $P_{Y}$ verifies axiom 6 , it can be furthermore assumed that $Z \subset \operatorname{RegP}(X)$ and that $Z$ is a regular curve. Let $v^{\prime}$ be a fixed discrete $k$-valuation of $K$ dominating $\mathcal{O}_{X, Z}$ with residue field a finite algebraic extension $k^{\prime}$ of $k(Z)$, and $\bar{v}$ be any discrete valuation of $k^{\prime}$. We denote by $v$ the composed valuation $v=v^{\prime} \circ \bar{v}$, with group $\mathbf{Z}_{\text {lex }}^{2}$. Let

$$
X=: X_{0} \leftarrow X_{1} \leftarrow \cdots \leftarrow X_{n} \leftarrow \cdots
$$

be that sequence of normalized blowing ups at closed points $z_{i} \in X_{i}$, where $z_{i}$ is the center of $v$. Since $P_{Y}$ verifies axiom 3 , it is sufficient to prove that $z_{i} \in \operatorname{RegP}_{Y}(X)$ for some $i \geq 0$. Let $I:=\mathcal{I O}_{X_{0}, z_{0}}$ and $f_{1} \in I$ be such that $v\left(f_{1}\right)=(a, b)$, where

$$
(a, b):=\min \{v(f) \mid f \in I\} .
$$

We claim that $I \mathcal{O}_{X_{i}, z_{i}}=\left(f_{1}\right)$ for some $i \geq 0$, which will complete the proof.

To prove the claim, let $I=\left(f_{1}, \ldots, f_{e}\right)$ be a minimal basis of $I$. By assumption, $I \mathcal{O}_{X_{0}, Z}=\left(f_{1}\right)$, hence there exists $g \in \mathcal{O}_{X_{0}, z_{0}}, v^{\prime}(g)=0$, such that $g f_{i} / f_{1} \in \mathcal{O}_{X_{0}, z_{0}}$ for $2 \leq i \leq e$. It can be assumed that $m:=\bar{v}(g)$ is minimal among all $g$ 's satisfying these properties. Assume that $e \geq 2$. Then $m \geq 1$ and $v\left(g f_{i} / f_{1}\right) \geq(0, m)$ by (4). Moreover $(t)=m_{z} \mathcal{O}_{X_{1}, z_{1}}$ factors out from $g$ and from $g f_{i} / f_{1}$ in $\mathcal{O}_{X_{1}, z_{1}}$. Since $0 \leq \bar{v}(g / t)<m$, we get a new invariant $\left(e_{1}, m_{1}\right)<_{\text {lex }}(e, m)$ w.r.t. $\mathcal{O}_{X_{1}, z_{1}}$. Therefore $e_{i}=1$ for some $i \geq 0$ and the claim is proved.

Finally, that $P_{Y}$ verifies axiom 5 is a consequence of the following lemma.

Lemma 3.3. (Refined local uniformization). Let $P$ be any regularity property verifying axioms 1 to 5 . Let $V$ be a $k$-valuation ring of $K$ and $X / k$ be a fixed projective model of $K$. There exists a projective model $Y / k$ of $K$ such that the center $y_{V} \in Y$ of $V$ satisfies $y_{V} \in \operatorname{RegP}(Y)$ and the rational map $Y \cdots \rightarrow X$ is everywhere defined.

Proof. Since $P_{X}$ verifies axiom 4 and $P$ verifies axiom 5 , there exists a projective model $X^{\prime} / k$ of $K$ such that $X^{\prime} \cdots \rightarrow X$ is defined at the center $x_{V}^{\prime} \in X^{\prime}$ and $x_{V}^{\prime} \in$ $\operatorname{RegP}\left(X^{\prime}\right)$. Let $Y$ be the closure of the graph of the rational map $X^{\prime} \cdots \rightarrow X$. Then $Y$ dominates $X$, and $Y$ is locally isomorphic to $X^{\prime}$ at $x_{V}^{\prime}$, so $Y$ satisfies the conclusion of the proposition.

Example 3.4. Let $P$ be any regularity property and $\mu \geq 1$ be an integer. For $X / k$ any projective model of $K$, define

$$
\operatorname{RegP}_{\mu}(X):=\left\{x \in \operatorname{RegP}(X): e_{X}(x) \leq \mu\right\},
$$

where $e_{X}(x) \geq 1$ denotes the multiplicity of $X$ at $x$. Note that $\operatorname{RegP}_{1}(X)=\operatorname{RegP}(X) \cap$ $X_{\text {reg }}$ with notations as in example 2.3.

Proposition 3.5. Let $P$ be any regularity property and $\mu \geq 1$ be an integer. If $P$ verifies axioms 1, 2 and 3 (resp. 1, 2, 3 and 6$)$, then $P_{\mu}$ also verifies axioms 1, 2 and 3 (resp. 1, 2, 3 and 6 ). If $X / k$ is any projective model of $K, x \in X$ is the generic point of an integral curve $Z \subset X$, and $z \in Z \cap \operatorname{RegP}_{\mu}(X)$ is such that $Z$ is stably $P$-permissible at $z$ and $e_{X}(z)=e_{X}(x)$, then $Z$ is stably $P_{\mu}$-permissible at $z$.

Proof. This is well known for axiom 1. Also, multiplicity does not increase by blowing up a point, nor by normalizing (theorem 14.8 [26]) and thus $P_{\mu}$ verifies axiom 2 if $P$ 
does. With assumptions as in the last statement of the proposition, we have

$$
e_{X^{\prime}}\left(z^{\prime}\right) \leq e_{E}\left(z^{\prime}\right) \leq \mu=e_{X}(x),
$$

where $\pi: X^{\prime} \rightarrow X$ is the blowing up along $Z, E:=\pi^{-1}(Z)$ and $z^{\prime} \in \pi^{-1}(z)$ is any point. Combining with (i) of axiom 2, this proves the last statement. With respect to axioms 3 and 6 , the proposition is a consequence of the following claim: let

$$
X=: X_{0} \leftarrow X_{1} \leftarrow \cdots \leftarrow X_{n} \leftarrow \cdots
$$

be that sequence of normalized blowing ups at points $z_{i-1} \in X_{i-1}$ such that $z_{0}:=z$ and for $1 \leq i \leq n, z_{i}$ maps to $z_{i-1}$ and belongs to the strict transform $Z_{i}$ of $Z$ in $X_{i}$. Then $e_{X_{n}}\left(z_{n}\right)=e_{X}(x)$ for all $n>>0$.

To prove this claim, it can be assumed that $\mu^{\prime}=e_{X_{n}}\left(z_{n}\right) \geq 2$ for all $n \geq 0$ without loss of generality. Consider the base change $\tilde{X}:=X \otimes \kappa(z)$ and pick a preimage $z^{\prime} \in \tilde{X}$ of $z, Z^{\prime}$ a reduced irreducible component of $Z \otimes \kappa(z)$ going through $z^{\prime}$, and $X^{\prime}$ a reduced irreducible component of $\tilde{X}$ containing $Z^{\prime}$. Without loss of generality, it can be assumed that $\left(\kappa(z), X^{\prime}, Z^{\prime}, z^{\prime}\right)=(k, X, Z, z)$ using basic properties of multiplicity (theorem 14.7 and $14.8[26]$ ).

Changing once again $k$ to a finite extension, it can be furthermore assumed that $K / k$ is separably generated. Let now $\pi: X \rightarrow \mathbf{A}_{k}^{3}$ be a generic projection around $z$. Since $\mu^{\prime} \geq 2, \pi$ factors through a hypersurface singularity $(Y, y) \subset \mathbf{A}_{k, 0}^{4}$ with $\mu^{\prime}=$ $e_{Y}(y)$ which is birational to $X$. In particular, $X$ is isomorphic to the normalization of $Y$ in a neighborhood of $y$. It can be furthermore assumed that $\pi$ is transverse to $Z$, i.e. that $e_{Y}(\pi(x))=e_{X}(x)$. We now define from (5) a sequence of maps:

$$
\begin{aligned}
& X=: X_{0} \leftarrow X_{1} \leftarrow \cdots \leftarrow X_{n}
\end{aligned}
$$

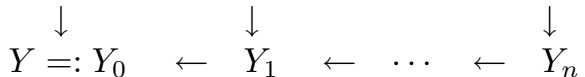

as follows, by induction on $i \geq 1$ : let $Y_{i} \rightarrow Y_{i-1}$ be the blowing up along the image $y_{i-1}$ of $z_{i-1}$ by the morphism $\pi_{i-1}: X_{i-1} \rightarrow Y_{i-1}$. If the ideal $m_{y_{i-1}} \mathcal{O}_{X_{i-1}, z_{i-1}}$ is a reduction of $m_{z_{i-1}}$ (which holds for $i=1$ ), both ideals have isomorphic normalized blowing up; on the other hand, the blowing up of $X_{i-1}$ along $m_{y_{i-1}} \mathcal{O}_{X_{i-1}, z_{i-1}}$ is then finite over $Y_{i}$, hence $X_{i}$ is isomorphic to the normalization of $Y_{i}$ above a neighborhood of $y_{i}$; finally $\mu^{\prime}=e_{X_{i}}\left(z_{i}\right) \leq e_{Y_{i}}\left(y_{i}\right) \leq e_{Y_{i-1}}\left(y_{i-1}\right)=\mu^{\prime}$ which implies that $m_{y_{i}} \mathcal{O}_{X_{i}, z_{i}}$ is a reduction of $m_{z_{i}}$.

Let $x_{i} \in X_{i}$ be the generic point of $Z_{i}$. Since $X_{i}$ is isomorphic to the normalization of $Y_{i}$ above a neighborhood of $y_{i}$, we have

$$
e_{X}(x)=e_{X_{i}}\left(x_{i}\right)=e_{Y_{i}}\left(\pi_{i}\left(x_{i}\right)\right) \leq e_{Y_{i}}\left(y_{i}\right)=e_{X_{i}}\left(z_{i}\right)=\mu^{\prime}
$$

for all $i \geq 0$. Since each $Y_{i} \rightarrow Y_{i-1}$ is the blowing up along $y_{i-1}$, it is well known that $e_{Y_{i}}\left(y_{i}\right)=e_{Y_{i}}\left(\pi_{i}\left(x_{i}\right)\right)$ for $i>>0$ and we get $e_{X_{i}}\left(z_{i}\right)=e_{X}(x)$ as required.

Example 3.6. [10] Assume that $k$ is algebraically closed, chark $=0$ and let $\delta \in$ $\operatorname{Der}_{k} K$ be a nonzero element. Let $X / k$ be a projective model of $K$ and $x \in X$ be a closed point such that $\mathcal{O}_{X, x}$ is a regular local ring. Given a germ of divisor with normal crossings $E_{x}=\operatorname{div}\left(u_{1} \cdots u_{e}\right)$ at $x, 0 \leq e \leq 3$, there exists an expression

$$
\delta=\phi_{x}\left(\sum_{i=1}^{e} f_{i, x} u_{i} \frac{\partial}{\partial u_{i}}+\sum_{j=e+1}^{3} f_{j, x} \frac{\partial}{\partial u_{j}}\right),
$$

with $\phi_{x} \in K, f_{1, x}, f_{2, x}, f_{3, x} \in \mathcal{O}_{X, x}$ and $\operatorname{dim}\left(\mathcal{O}_{X, x} /\left(f_{1, x}, f_{2, x}, f_{3, x}\right)\right) \leq 1$. 
If $x \in X$ is any closed point, we say that $\delta$ is log-elementary at $x$ w.r.t. $E_{x}$ if $x$ is a regular point of $X$ and

$$
\nu_{x}\left(\delta, E_{x}\right):=\min \left\{\operatorname{ord}_{x} f_{i, x}, 1 \leq i \leq 3\right\} \leq 1 .
$$

We say that $\delta$ is log-elementary at $x$ if there exists a germ of divisor with normal crossings $E_{x}$ at $x$ such that $\delta$ is log-elementary at $x$ w.r.t. $E_{x}$. These notions extend to arbitrary (not necessarily closed) points: $\delta$ is log-elementary at a point $x$ if it is at a general point of $\overline{\{x\}}$.

Let $P$ be any regularity property. We now define the regularity property $P_{\delta}$ as follows: for any projective model $X / k$ of $K$, let

$$
\operatorname{RegP}_{\delta}(X):=\operatorname{RegP}(X) \cap\{x \in X \mid \delta \text { is pseudo - elementary at } x\} .
$$

The following proposition is a consequence of the preparatory results in chapter I of $[7]$.

Proposition 3.7. Assume that $k$ is algebraically closed, chark $=0$ and let $\delta \in \operatorname{Der}_{k} K$ be a nonzero element. If $P$ verifies axioms 1, 2 and 3 (resp. 1, 2, 3 and 6), then $P_{\delta}$ also verifies axioms 1, 2 and 3 (resp. 1, 2, 3 and 6).

Proof. Although the definition of log-elementary singularities is pointwise, the exceptional divisor $E_{x}$ can be taken to be locally constant on a given variety $X$ and this proves that axiom 1 is verified by $P_{\delta}$. Note that proposition I.3.4.9 of [7] allows to make blowing up along stably $P_{\delta}$-permissible compatible with $E_{x}$.

Axioms 2 and 3 are verified by [7], remark I.3.4.5 and proposition I.3.4.9 when $P=P_{\text {reg }}$ and this immediately extends to arbitrary $P$ verifying axioms $1,2,3$. So is axiom 6 , in view of the corresponding result for $P_{\mu}$ in proposition 3.5 with $\mu:=1$.

To state our third family of applications, we need the following definition.

Definition 3.8. Let $P$ be any regularity property. Let $X, Y$ be projective models of $K, Y$ normal, and $x \in Y$. The birational map $\eta: X \cdots \rightarrow Y$ is said to be a $P$-regular sequence at $x$ if the following holds:

(i) $\eta$ is defined at $x$ and $y:=\eta(x) \in \operatorname{Reg} \mathrm{P}(Y)$.

(ii) there exists a sequence of projective birational morphisms

$$
Y=: Y_{0} \leftarrow Y_{1} \leftarrow \cdots \leftarrow Y_{n},
$$

where for each $i, 1 \leq i \leq n, Y_{i} \rightarrow Y_{i-1}$ is the normalized blowing up along a reduced and irreducible closed subset $Z_{i-1} \subset Y_{i-1}, \operatorname{dim} Z_{i-1} \leq 1$, whose generic point belongs to $\operatorname{Reg} \mathrm{P}\left(Y_{i-1}\right)$, and such that

(a) the rational map $\eta: X \cdots \rightarrow Y_{n}$ is defined and locally an isomorphism at $x$;

(b) for $1 \leq i \leq n, Z_{i-1}$ is stably $P$-permissible at the point $\pi_{i}(\eta(x))$, where $\pi_{i}$ is the composed map $Y_{n} \rightarrow Y_{i-1}$.

In case $P=P_{\text {reg }}$ is the usual regularity property, we will use the classical terminology of "monoidal sequence" instead of " $P_{\text {reg-regular sequence": in this case each }}$ $Z_{i-1}$ appearing in (ii) of the definition can be taken to be either a closed point, or an irreducible curve which contains and is regular at $\pi_{i}(\eta(x))$. From the above definition of $P$-regular sequences we associate the following regularity property $P_{R S(Y)}$ to any regularity property $P$ having a globally $P$-regular model $Y$. 
Definition 3.9. Let $P$ be a regularity property and $Y$ be a normal projective model of $K$ such that $\operatorname{RegP}(Y)=Y$.

We define a regularity property $P_{R S(Y)}$ as follows: for any birational projective model $X / k$ of $K, \operatorname{RegP}_{R S(Y)}(X)$ is the set of points $x \in X$ such that the birational map $\eta: X \cdots \rightarrow Y$ is a $P$-regular sequence at $x$.

Proposition 3.10. Let $P$ be any regularity property and $Y$ be a normal projective model of $K$ such that $\operatorname{RegP}(Y)=Y$.

Assume that $P$ veryfies axioms 1,2 and 3. Then $P_{R S(Y)}$ also verifies axioms 1, 2 and 3.

Proof. Let $X / k$ be any projective model of $K$ and $C \subset X$ be an integral curve whose generic point is normal and belongs to $\operatorname{RegP}(X)$. Since $P$ verifies axioms 1,2 and 3, the set

$$
V_{C}:=\{z \in C \mid C \text { is stably permissible at } z\}
$$

is a nonempty open set of $C$ (cf. section 2, comments after the definition of axiom 3 ).

Let now $x \in \operatorname{RegP}_{R S(Y)}(X)$ and

$$
Y=: Y_{0} \leftarrow Y_{1} \leftarrow \cdots \leftarrow Y_{n}
$$

be a sequence of normalized blowing ups at integral varieties $Z_{i-1}, 1 \leq i \leq n$ given by (ii) of definition 3.8 and keep notations as in there. For each $i$, we may choose a nonempty open set $U_{i-1} \subseteq Y_{i-1}$ such that $\pi_{i}(\eta(x)) \in U_{i-1}$ and $Z_{i-1}$ is stably $P$ permissible at all points of $\bar{Z}_{i-1} \cap U_{i-1}$. Then $U:=\pi_{1}^{-1}\left(U_{0}\right) \cap \cdots \cap \pi_{n}^{-1}\left(U_{n-1}\right) \subseteq Y_{n}$ is Zariski open and $\eta(x) \in U$. After possibly restricting $U$, it can be assumed that $\eta$ is defined and is an isomorphism above $U$. Let now $x^{\prime} \in \eta^{-1}(U)$. Then $Y_{i} \rightarrow Y_{i-1}$ is an isomorphism at $\pi_{i+1}\left(\eta\left(x^{\prime}\right)\right)$ if and only if $\pi_{i}\left(\eta\left(x^{\prime}\right)\right) \notin Z_{i-1}$. Therefore $x^{\prime} \in \operatorname{RegP}_{R S(Y)}(X)$ and an associated sequence satisfying the conditions of (ii.b) of definition 3.8 is obtained by erasing from (9) those $Y_{i}^{\prime}$ 's such that $\pi_{i}\left(\eta\left(x^{\prime}\right)\right) \notin Z_{i-1}$. This proves that $\operatorname{Reg} \mathrm{P}_{R S(Y)}(X)$ is Zariski open, hence $\operatorname{Reg} \mathrm{P}_{R S(Y)}$ verifies axiom 1 .

Since $\operatorname{RegP}_{R S(Y)}(X)$ is normal by (ii) of definition 3.8 ( $Y$ being normal), $\operatorname{Reg} \mathrm{P}_{R S(Y)}$ verifies (i) of axiom 2. Now (ii) of axiom 2 is tautological if $x$ is a closed point or is a consequence of the openness of the set $V_{C}$ defined at the beginning of this proof if $x$ is the generic point of an integral curve $C$.

To prove that axiom 3 is also verified by $P_{R S(Y)}$, let $X / k$ be any normal projective model of $K, C \subset X$ be an integral curve whose generic point belongs to $\operatorname{RegP}_{R S(Y)}(X)$ and $z \in C$. By definition of $P_{R S(Y)}, C$ is stably $P_{R S(Y)}$-permissible at $z$ if and only if $C$ is stably $P$-permissible at $z$. Moreover, if $\pi: X_{1} \rightarrow X$ is the normalized blowing up along $z, C_{1} \subset X_{1}$ the strict transform of $C$, and $z_{1} \in C_{1}$ satisfies $\pi\left(z_{1}\right)=z$, then $z_{1} \in \operatorname{RegP} P_{R S(Y)}\left(X_{1}\right)$ since $P$ verifies axiom 2 . Therefore $P_{R S(Y)}$ verifies axiom 3 if $P$ does.

Another way of constructing regularity properties is by conjunction.

Proposition 3.11. Let $P_{1}$ and $P_{2}$ be two regularity properties and let $P$ be the conjunction of $P_{1} \wedge P_{2}$, i.e.

$$
\operatorname{RegP}(X):=\operatorname{RegP}_{1}(X) \cap \operatorname{RegP}_{2}(X)
$$

for any projective model $X / k$ of $K$. If each of $P_{1}$ and $P_{2}$ verifies any of axioms 1 , 2 and 3 (resp. verifies axioms 3 and 6$)$, so does $P$ (resp. $P$ verifies axioms 3 and 6$)$.

Proof. This is a direct consequence of the definition of axioms 1, 2 and 3 and 6. 
Remark: propositions 3.10 and 3.11 need not extend to axioms 4 and 5 in general. The reason is that the definition of these axioms involves no blowing up procedure to build up $\tilde{X}$ (axiom 4) or $X$ (axiom 5) independently of $P$. See however the next section for results about axioms 4 (propositions 4.1 and 4.2) and 6 (proposition 4.7) when $P_{1}$ and $P_{2}$ are refinements of the usual regularity property.

\section{REFinements of the USUAL REgUlarity PROPERTy.}

In this section, we assume that $P$ is a refinement of the usual regularity property, i.e. $\operatorname{Reg} \mathrm{P}(X) \subseteq X_{\text {reg }}$ for any projective model $X / k$ of $K$.

4.1. Principalization of ideals. We produce various criteria for a regularity property to verify axiom 4 . The main difficulty here is that neither of the two standard algorithms for principalizing ideals on a regular threefold (i.e. Hironaka's [19] and Abhyankar's [3]) does the job. The reason is that regular curves which are permissible for these algorithms need not be $P$-permissible in the sense of definition 2.2. See also the last section on open problems.

That the usual regularity property $P=P_{\text {reg }}$ verifies axiom 4 in all characteristics is proved in proposition 4.2 of [12]. We sum up below the algorithm given in loc.cit. One immediately reduces to the idealistic exponent formulation in proposition 4.3 of [12]: given $\mathcal{E}=(\mathcal{I}, \mu)$, an idealistic exponent on $U:=\operatorname{RegP}(X)$, with

$$
\operatorname{dim}\left(\operatorname{Supp}\left(\mathcal{O}_{X} / \mathcal{I}\right)\right) \leq 1,
$$

there exists a finite composition of blowing ups

$$
U=: U(0) \leftarrow U(1) \leftarrow \cdots \leftarrow U(n)
$$

with stably $P$-permissible centers (i.e. regular in the usual sense) mapping to $V(\mathcal{I})$ such that the singular locus $\Sigma(n)$ of the transform $\mathcal{E}(n)$ of $\mathcal{E}$ in $U(n)$ is empty.

Let $\mathcal{E}(i):=(\mathcal{I}(i), \mu)$ be the transform of $\mathcal{E}$ in $U(i), \Sigma(i)$ its singular locus. The existence of (11) with the required properties is proved by applying the following algorithm, denoted (A) in the sequel, and showing that it eventually stops:

1. If $\Sigma(i)$ has an irreducible component $C$ of dimension one which is singular (in the usual sense), let $U(i+1) \rightarrow U(i)$ be the blowing up along a singular point of $C$. Otherwise go to 2 .

2. If $\Sigma(i)$ has (regular) components of dimension one which do not intersect transversally, or three such components going through one and the same point, let $U(i+1)$ be the blowing up of $U(i)$ along any such intersection point. Otherwise go to 3 .

3. If two components of dimension one of $\Sigma(i)$ do intersect, let $U(i+1)$ be the blowing up of $U(i)$ along any such intersecting curve. Otherwise go to 4 .

4. If $\Sigma(i) \neq \emptyset$, let $X(i+1)$ be the blowing up of $X(i)$ along any of its connected components. If $\Sigma(i)=\emptyset$, stop the algorithm.

The following proposition gives a criterion for axiom 4 to be verified.

Proposition 4.1. Let $P$ be a regularity property which is a refinement of the usual regularity property and verifies axioms 1,2 and 3. Assume that the following holds: for any projective model $X / k$ of $K$, for any $x \in X$ with $\operatorname{dim} \mathcal{O}_{X, x}=2$ and for any $z \in Z \cap \operatorname{RegP}(X)(Z:=\overline{\{x\}})$ such that $Z$ is regular at $z$ (in the usual sense), $Z$ is $P$-permissible at $z$. Then $P$ verifies axiom 4 . 
Proof. The assumption implies that any blowing up center $Z(i)$ on some $U(i), i \geq 0$ which is permissible for the idealistic exponent $\mathcal{E}(i)$ is actually $P$-permissible at each $z(i) \in Z(i)$. So the proof is the same as for $P=P_{\text {reg. }}$.

Proposition 4.2. Assume that $k$ is algebraically closed and chark $=0$ and let $\delta \in$ $\operatorname{Der}_{k} K$ be a nonzero element. Let $P$ be a regularity property satisfying the assumptions of proposition 4.1. Then $P_{\delta}$ verifies axiom 4.

Proof. With notations as in the beginning of this section, let $\mathcal{E}=(\mathcal{I}, \mu)$ be an idealistic exponent on $U:=\operatorname{RegP}(X)$ satisfying (10). It is sufficient to prove the existence of a sequence (11) of blowing ups with stably $P_{\delta}$-permissible centers such that the singular locus $\Sigma(n)$ of the transform $\mathcal{E}(n)$ of $\mathcal{E}$ in $U(n)$ is empty. We will assume familiarity with embedded resolution procedures in regular threefolds in this proof, e.g. [19] or proposition 4.2 of [12] (which are characteristic free), as well as with the definitions and preliminary results in chapter I of [7].

Let $x \in U$. By definition of log-elementary singularities (example 3.6), there exists a germ of normal crossings divisor $E_{x}$ at $x$ such that $\delta$ is expressed locally et $x$ by formula (7) with property (8). In particular, there exists an open neighborhood $V_{x}$ of $x$ and a normal crossings divisor $E$ on $V_{x}$ such that $\delta$ is log-elementary at each $y \in V_{x}$ w.r.t. the stalk $E_{y}$ of $E$ at $y$. One easily deduces that there exists a finite open covering $V_{1}, \ldots, V_{s}$ of $U$ and a normal crossings divisor $E_{j}$ on $V_{j}$ for each $j$, $1 \leq j \leq s$ such that the following holds: for any $x \in U$ and any $j, 1 \leq j \leq s$ with $x \in V_{j}, \delta$ is log-elementary at $x$ w.r.t. $E_{j, x}$. We fix such an open covering to begin with and first reduce to the case $s=1$.

Definition 4.3. With notations as above, a blowing up center $Y(i) \subset U(i)$ is said to be $\left(\mathcal{E}(i), P_{\delta}\right)$-permissible at a point $x(i) \in Y(i)$ if it is stably $P$-permissible, permissible for $\mathcal{E}(i)=(\mathcal{I}(i), \mu)$, the transform of $\mathcal{E}$ in $U(i)$, and "permissible for $\delta$ adapted to the total transform of each $E_{j}, 1 \leq j \leq s$ " at $x(i)$ in the sense of [7] I.3.4.4. A sequence of blowing ups

$$
U=: U(0) \leftarrow U(1) \leftarrow \cdots \leftarrow U(n)
$$

is said to be an $\left(\mathcal{E}, P_{\delta}\right)$-sequence if for each $i, 0 \leq i \leq n-1$, the blowing up center $Y(i) \subset U(i)$ is $\left(\mathcal{E}(i), P_{\delta}\right)$-permissible at each of its points.

In the above definition, note that $Y(i)$ is stably $P$-permissible iff $Y(i)$ is regular, since $P$ satisfies the assumptions of proposition 4.1. Also $Y(i)$ is permissible for $\mathcal{E}(i)$ iff $Y(i) \subseteq \Sigma(i)$. The definition of permissibility in [7] I.3.4.4 is a refinement of $P_{\delta}$-permissibility in definition 2.2 designed to get compatibility of blowing ups with normal crossings divisors. An immediate consequence of [7] proposition I.3.4.9 is that the strict transform $Y(i+j)$ of any curve $Y(i) \subset \Sigma(i)$ can be made $\left(\mathcal{E}(i+j), P_{\delta}\right)$ permissible after blowing up $j \geq 0$ many times along closed points.

Let $\pi(i): U(i) \rightarrow U$ be the induced morphism. Given an $\left(\mathcal{E}, P_{\delta}\right)$-sequence, we let

$$
E_{j}(i):=\pi(i)^{-1}\left(E_{j}\right)_{\text {red }} \subset V_{j}(i):=\pi(i)^{-1}\left(V_{j}\right) .
$$

In particular, the following holds for each $i, 0 \leq i \leq n$ : $\operatorname{RegP}_{\delta}(U(i))=U(i) ; \pi(i)$ is an isomorphism away from $\Sigma$; for each $j, 1 \leq j \leq s, E_{j}(i)$ is a normal crossings divisor on $V_{j}(i)$ such that $\delta$ is log-elementary at each $x(i) \in V_{j}(i)$ w.r.t. the stalk of $E_{j}(i)$ at $x(i)$. Moreover, $Y(i)$ has normal crossings with $E_{j}(i)$ for each $i, j, 0 \leq i \leq n-1$, $1 \leq j \leq s$.

Lemma 4.4. There exists an $\left(\mathcal{E}, P_{\delta}\right)$-sequence (12) such that $\pi(n)(\Sigma(n))$ is a finite set. 
Proof. Let $C \subset \Sigma$ be an irreducible curve. We apply the following algorithm (B) for $i \geq 0$ :

1. If $\Sigma(i)$ has an irreducible component $C(i)$ with $\pi(i)(C(i))=C$, and a point $x(i) \in C(i)$ at which $C(i)$ is not $\left(\mathcal{E}, P_{\delta}\right)$-permissible, let $U(i+1) \rightarrow U(i)$ be the blowing up along $x(i)$. Otherwise go to 2 .

2. If $\Sigma(i)$ has an irreducible component $C(i)$ with $\pi(i)(C(i))=C$, let $U(i+1) \rightarrow U(i)$ be the blowing up along $C(i)$ and go back to step 1. Otherwise stop.

To prove the lemma, it is thus sufficient to prove that algorithm (B) stops, since $\Sigma$ has finitely many irreducible components of dimension one. As mentioned right after definition 4.3, one reaches step 2 of algorithm (B) after finitely many point blowing ups. If the algorithm does not stop, we get an infinite quadratic sequence of two dimensional regular local rings

$$
\mathcal{O}_{U\left(i_{1}\right), C\left(i_{1}\right)}<\mathcal{O}_{U\left(i_{2}\right), C\left(i_{2}\right)}<\cdots<\mathcal{O}_{U\left(i_{n}\right), C\left(i_{n}\right)}<\cdots,
$$

with $C\left(i_{n}\right) \subseteq \Sigma\left(i_{n}\right)$ for each $n \geq 1$ : a contradiction by e.g. [34] appendix 5 , theorem 3 and (E) on p. 391.

Choosing $j=1$ at each of the finitely many points in $\pi(n)(\Sigma(n))$ and working consecutively on each connected component of $\Sigma(n)$, we get:

Corollary 4.5. With notations as above, it can be assumed that $s=1$ and $\Sigma \subset E_{1}$.

In view of this corollary, we simplify our notations and write $E$ instead of $E_{1}$, take $V_{1}=U$. For $x(i) \in \Sigma(i)$, let $m(x(i)), 1 \leq m(x(i)) \leq 3$, be the number of irreducible components of $E(i)_{x(i)}$ going through $x(i)$. Recall the standard definition of the directrix vector space $T_{x(i)}$ and $\tau(x(i)):=\operatorname{dim}_{k} T_{x(i)}$ of $\mathcal{E}(i)$ (cf. [12] section 4).

Lemma 4.6. There exists an $\left(\mathcal{E}, P_{\delta}\right)$-sequence (12) such that the following holds.

(i) $\Sigma(n) \subset E(n)$ is a union of isolated points and projective lines and has normal crossings with $E(n)$. Any two distinct irreducible components of $\Sigma(n)$ meet at at most one point, any three of them do not meet.

(ii) for each $x(n) \in \Sigma(n)$, there exists $Z_{x(n)} \in T_{x(n)}$ which has normal crossings with $E(n)$ at $x(n)$. In particular $m(x(n)) \leq 2$.

Moreover, (i) and (ii) are preserved by any further $\left(\mathcal{E}, P_{\delta}\right)$-permissible blowing ups.

Proof. If $\pi(1): U(1) \rightarrow U$ is the blowing up along an $\left(\mathcal{E}, P_{\delta}\right)$-permissible center $Y \subset U$, with exceptional divisor $F$, and $x \in Y$, we have $\pi(1)^{-1}(x) \cap \Sigma(1)=\emptyset$ except in the following situations:

(a) $Y=x$ is a closed point, $\tau(x)=2$ and $F \cap \Sigma(1)$ is the isolated point $x(1)$ corresponding to the projective space associated with $T_{x}$ which has $\tau(x(1)) \geq 2$.

(b) $Y$ is a curve, $\tau(x)=1$ and $F \cap \Sigma(1)$ either is a finite set, or is a regular curve mapping isomorphically to $Y$.

(c) $Y=x$ is a closed point, $\tau(x)=1$ and $F \cap \Sigma(1)$ is contained in the projective line $L_{x} \subset F$ associated with $T_{x}$.

In view of these facts and of lemma 4.4, we can achieve $\Sigma(n)$ to be a union of isolated points and projective lines. Applying steps 1 and 2 of algorithm (A), we can furthermore assume that any two distinct projective lines in $\Sigma(n)$ intersect transversally on $E(n)$ and at at most one point, any three of them do not have one and the same intersection point. Note that this is preserved by any further $\left(\mathcal{E}, P_{\delta}\right)$-sequence. 
Without loss of generality, we let $n=0$ in the sequel. The last statement in the lemma is an easy consequence of (a)-(c). Finally, (i) is achieved from (ii) by a finite composition of blowing ups at closed points performing embedded resolution of $\Sigma(n) \cup M_{2}(n)$, where

$$
M_{2}(n):=\{x(n) \in U(n): m(x(n)) \geq 2\} .
$$

There hence remains to prove (ii). We will repeatedly use the following fact: if $x \in \Sigma$ is a closed point with r.s.p. $\left(u_{1}, u_{2}, u_{3}\right)$, then

$$
Y=V\left(u_{1}, u_{2}\right) \subseteq \Sigma \Longrightarrow T_{x} \subseteq<U_{1}, U_{2}>
$$

Now consider consecutively the following cases:

Case 1. $\tau(x)=2$ and $x$ is an isolated point of $\Sigma$. By (a), blowing up $x$ gives either $F \cap \Sigma(1)=\emptyset$, or $F \cap \Sigma(1)=\{x(1)\}$ with $\tau(x(1)) \geq 2$. Iterating, the process will stop after finitely many blowing ups at closed points because $x$ is an isolated point of $\Sigma$.

Case 1'. $\tau(x)=2$ and $x$ is not an isolated point of $\Sigma$. Since $\tau(x)=2, \Sigma$ is locally at $x$ an irreducible curve $C_{x}$ by (13). Blowing up gives $\pi(1)^{-1}(x) \cap \Sigma(1)=\emptyset$ unless ( $Y=x$ and $\Sigma(1)$ coincides with the strict transform $C_{x}(1)$ of $C_{x}$ in a neighborhood of $F$ ); then $\tau(x(1)) \geq 2$, where $F \cap C_{x}(1)=:\{x(1)\}$ by (a). Iterating, it can therefore be assumed w.l.o.g. that $C_{x}$ is $\left(\mathcal{E}, P_{\delta}\right)$-permissible at $x$.

Assume now that $\tau(x)=1$. Pick a r.s.p. $\left(u_{1}, u_{2}, u_{3}\right)$ at $x$ such that $E_{x}=$ $\operatorname{div}\left(u_{1} \cdots u_{e}\right)$, where $e=m(x)$. Let $T_{x}=:<\alpha_{1} U_{1}+\alpha_{2} U_{2}+\alpha_{3} U_{3}>$. Let $\epsilon(x):=0$ if $\alpha_{j} \neq 0$ for some $j \geq e+1$; otherwise define:

$$
\epsilon(x):=\sharp\left\{j: 1 \leq j \leq e \text { and } \alpha_{j} \neq 0\right\} \geq 1 .
$$

Note that $x$ satisfies (ii) if and only if $\epsilon(x)=0$.

Case 2. $\epsilon(x)=3$. If $x$ is not an isolated point of $\Sigma$, any component of $\Sigma$ is locally at $x$ an irreducible curve $C$, tangent to the line $L:=V\left(\alpha_{1} u_{1}+\alpha_{2} u_{2}, u_{3}\right)$ up to permuting indices by (13). In any case, we get (ii) with $n=1$ by blowing up $x$ by (c).

Case 3. $\epsilon(x)=2$. If $x$ is not an isolated point of $\Sigma$, any component of $\Sigma$ is locally at $x$ either (tangent to the line $L:=V\left(\alpha_{1} u_{1}+\alpha_{2} u_{2}, u_{3}\right)$ up to permuting indices with $e=3)$, or tangent to the line $L_{1}:=V\left(u_{1}, u_{2}\right)$ by (13). If $Y=C$ is a curve, then $C=L_{1}$ and (ii) holds with $n=1$ above $x$ by (b). Otherwise, blowing up $x$ gives (ii) with $n=1$ above $x$ except possibly at the point $x(1)$ on the strict transform of $L_{1}$. The point $x(1)$ is either in one of cases 1,1 ' or 2 , or again in case 3 and we can iterate. Then we either get (ii) for some $n \geq 1$ above $x$ or $L_{1} \subseteq \Sigma$ and its strict transform $L_{1}(n)$ at $x(n)$ is $\left(\mathcal{E}(n), P_{\delta}\right)$-permissible at $x(n)$. In any case, we get (ii) above $x$ except possibly at $x(n)$.

Case 4. $\epsilon(x)=1$. Let $R:=\{x \in \Sigma: \tau(x)=\epsilon(x)=1\}$. Then $R$ is a closed subset of $\Sigma$ and the component $D_{x}:=\operatorname{div}\left(u_{i}\right)$ of $E_{x}$ such that $T_{x}=k . U_{i}$ is constant when $x$ varies in a connected component of $R$. If $x \in R$ and $\pi(1): U(1) \rightarrow U$ is the blowing up along $x$, then $F \cap \Sigma(1)$ is contained in the strict transform of $D_{x}$ by (c). Suppose that there exists an irreducible curve $C \subseteq D \cap \Sigma$ such that $C \nsubseteq M_{2}:=M_{2}(0)$. Applying algorithm (B) to $C$ finitely many times and taking into account the analysis of cases 1 ' and 3 when $Y=C$, we reduce to the case $D \cap \Sigma \subseteq M_{2}$. In particular, we may 
assume w.l.o.g. the following: there exists an irreducible component $D \subseteq E$ such that $x \in R \Longrightarrow x \in D \cap M_{2}$ and $D_{x}=\operatorname{div}\left(u_{1}\right)$ locally at $x$.

We now use classical results on principalization of ideals. If $x \in R$ and $g \in \mathcal{I}_{x}$, there is a unique expansion

$$
g=g_{0} u_{1}^{\mu}+\sum_{i=1}^{\mu} g_{i} u_{1}^{\mu-i},
$$

with $g_{0} \in k\left[\left[u_{1}, u_{2}, u_{3}\right]\right] ; g_{i} \in k\left[\left[u_{2}, u_{3}\right]\right]$ and $\operatorname{ord}_{\left(u_{2}, u_{3}\right)} g_{i}>i$ for $1 \leq i \leq \mu$. Let $\pi(1): U(1) \rightarrow U$ be the blowing up along $x$. The ideal

$$
c\left(\mathcal{I}_{x}\right):=\left(\left\{g_{i}^{\mu ! / i}: 1 \leq i \leq \mu, g \in \mathcal{I}_{x}\right\}\right),
$$

transforms by the following rule at $x(1) \in F \cap \Sigma(1)$ : with obvious notations, we have $c\left(\mathcal{I}(1)_{x(1)}\right)=u_{j}^{-\mu !} c\left(\mathcal{I}_{x}\right)$, where $\left(u_{j}\right)$ is the ideal of $F=\pi(1)^{-1}(x)$ at $x(1), j=2$ or $j=3$. By embedded resolution of curves in regular surfaces, [17] proposition V.3.9, it can be furthermore assumed that for each $x \in R$,

$$
x \in R \Longrightarrow c\left(\mathcal{I}_{x}\right)=\left(u_{2}^{a_{2, x}} u_{3}^{a_{3, x}}\right)
$$

with $a_{2, x}, a_{3, x} \in \mathbf{N},\left(a_{2, x}+a_{3, x}\right) / \mu$ ! $>1$. By (14), we have for $x \in R$ :

$$
m(x)=2 \Longrightarrow 0 \leq a_{3, x} / \mu !<1 \text {. }
$$

If $Y \subset D \cap M_{2}$ is an irreducible curve meeting $R$, we let

$$
a_{Y}:=a_{i, x}, x \in Y \cap R \text { arbitrary and } Y=V\left(u_{1}, u_{i}\right) \text { locally at } x .
$$

If $Y \subset D \cap M_{2}$ is an irreducible curve not meeting $R$, we simply let $a_{Y}:=0$. If $x \in R$, we let

$$
\alpha(x):=\max \left\{a_{2, x} / \mu !, a_{3, x} / \mu !\right\}, c(x):=\left(a_{2, x}+a_{3, x}\right) / \mu ! .
$$

Case 4.1. $x \in R$ is an isolated point of $\Sigma$. This is equivalent to $\alpha(x)<1$. Any $x(1) \in F \cap \Sigma(1)$ is also an isolated point of $\Sigma(1)$, and $c(x(1))<c(x)$ if $x(1) \in R(1)$. Iterating the argument and taking into account the analysis of cases 1,2 and 3 , we may assume furthermore that $\Sigma$ has equal dimension one.

We now need use the specific properties of log-elementary singularities of vector fields. Recall from $(8)$ the definition of $\nu_{x}\left(\delta, E_{x}\right)$. We let

$$
\Sigma_{0}:=\left\{x \in \Sigma: \nu_{x}\left(\delta, E_{x}\right)=0\right\}, \Sigma_{1}:=\left\{x \in \Sigma: \nu_{x}\left(\delta, E_{x}\right)=1\right\}=\Sigma \backslash \Sigma_{0} .
$$

Note that $\Sigma_{0}$ is open in $\Sigma$. The following is the content of I.3.4.1 (with $\nu_{x}\left(\delta, E_{x}\right) \leq 1$ in our situation), I.3.4.4 (with $m(x) \geq 2$ if $x \in \Sigma$ in our situation) and I.3.4.6 of [7]: if $Y \subseteq \Sigma$ is an irreducible curve, then

$$
Y \text { is }\left(\mathcal{E}, P_{\delta}\right) \text { - permissible at } x \in Y \Leftrightarrow x \in \Sigma_{0} \text { or } Y \subseteq \Sigma_{1} .
$$

Moreover, if a center $Y$ is $\left(\mathcal{E}, P_{\delta}\right)$-permissible at some point $x \in Y$ and $\pi(1): U(1) \rightarrow$ $U$ is the blowing up along $Y$ and $x(1) \in \pi(1)^{-1}(x)$, then

$$
\nu_{x(1)}\left(\delta, E(1)_{x(1)}\right) \leq \nu_{x}\left(\delta, E_{x}\right) .
$$

The vector field $\delta$ induces a vector field $\bar{\delta}$ by projection on $D$. Given a local expansion at $x$

$$
\delta=\phi_{x}\left(\sum_{i=1}^{e} f_{i, x} u_{i} \frac{\partial}{\partial u_{i}}+\sum_{j=e+1}^{3} f_{j, x} \frac{\partial}{\partial u_{j}}\right)
$$


$\phi_{x} \in K, f_{1, x}, f_{2, x}, f_{3, x} \in \mathcal{O}_{U, x}, \operatorname{dim}\left(\mathcal{O}_{U, x} /\left(f_{1, x}, f_{2, x}, f_{3, x}\right)\right) \leq 1$ and $\nu_{x}\left(\delta, E_{x}\right)=$ $\min \left\{\operatorname{ord}_{x} f_{i}, 1 \leq i \leq 3\right\} \leq 1$, denote $\bar{f}_{j, x}, 1 \leq j \leq 3$ the image of $f_{j, x}$ in $\mathcal{O}_{D, x}$. Then $\bar{\delta}$ is given at $x$ by

$$
\bar{\delta}=\sum_{i=2}^{e} \bar{f}_{i, x} u_{i} \frac{\partial}{\partial u_{i}}+\sum_{j=e+1}^{3} \bar{f}_{j, x} \frac{\partial}{\partial u_{j}},
$$

where we still denote $u_{2}, u_{3}$ the images in $\mathcal{O}_{D, x}$ of the corresponding parameters of $\mathcal{O}_{U, x}$. If $U(n) \rightarrow U$ is a finite composition of blowing ups at closed points and $x(n) \in D(n)$ is a closed point with r.s.p. $\left(u_{1, x(n)}, u_{2, x(n)}, u_{3, x(n)}\right)$, we write similarly

$$
\delta=\phi_{n, x(n)}\left(\sum_{i=1}^{e} f_{i, x(n)} u_{i, x(n)} \frac{\partial}{\partial u_{i, x(n)}}+\sum_{j=e+1}^{3} f_{j, x(n)} \frac{\partial}{\partial u_{j, x(n)}}\right),
$$

where $e=m(x(n)), D(n)_{x(n)}=\operatorname{div}\left(u_{1, x(n)}\right)$ and let

$$
\bar{J}_{x(n)}:=\left(\bar{f}_{2, x(n)}, \bar{f}_{3, x(n)}\right), \bar{K}_{x(n)}:=\bar{J}_{x(n)}+\left(\bar{f}_{1, x(n)}\right) .
$$

Using two-dimensional results on resolution of vector fields [28], there exists a finite composition of blowing ups at closed points $U(n) \rightarrow U$ such that the following holds: for each $x(n) \in D(n) \cap \Sigma(n)$, exactly one of the following holds:

$(\alpha) x(n) \notin \Sigma_{1}(n)$, or

( $\beta) \bar{J}_{x(n)}=\left(u_{2, x(n)}^{b_{2, x(n)}} u_{3, x(n)}^{b_{3, x(n)}}\right)$ is a monomial (possibly zero) ideal, where $b_{3, x(n)}=0$ if $m(x(n))=2$, or

$(\gamma) \bar{J}_{x(n)}=\left(u_{2, x(n)}^{\left.b_{2, x(n)}\right)} \bar{J}_{x(n)}^{\prime}\right.$, with $m_{x(n)}=2$ and $\left(u_{2, x(n)}\right)+\bar{J}_{x(n)}^{\prime}=\left(u_{2, x(n)}, \bar{f}_{3, x(n)}\right)=$ $\left(u_{2, x(n)}, u_{3, x(n)}\right)$.

Moreover, $((\alpha)$ or $(\beta))$ is preserved by further blowing ups at closed points. Any (finite or infinite) chain of points $x(n), x(n+1), \ldots$ satisfying $(\gamma)$ belongs to the strict transform of a regular, possibly formal curve on $D(n)$. Therefore $x(n+m) \notin \Sigma(n+m)$ for some $m \geq 0$, and this implies that there exists $n_{1} \geq 0$ such that $((\alpha)$ or $(\beta))$ holds for all $n \geq n_{1}$. We take $n_{1}=0$ w.l.o.g. For $x \in \Sigma_{1}$, let $\pi(1): U(1) \rightarrow U$ be the blowing up along $x$ and $x(1) \in F \cap \Sigma(1)$. We get the following transformation law from the blowing up formulæ [7] I.2.2.5:

$$
\bar{K}_{x(1)}=\left(u_{j}^{-\epsilon} \bar{f}_{1, x}\right)+\bar{J}_{x(1)},
$$

where $\left(u_{j}\right)$ is the ideal of $F=\pi(1)^{-1}(x)$ at $x(1), j=2$ or $j=3$, and $\epsilon=0$ (resp. $\epsilon=1)$ if $\left(m(x)=2\right.$ and $\left.\operatorname{ord}_{x} f_{3, x}=1\right)$ (resp. otherwise).

Using once more the blowing up formulæ [7] I.2.2.5 and controlling the order of the transform of $\bar{K}_{x}$ by (20), there exists a finite composition of blowing ups at closed points $U\left(n_{2}\right) \rightarrow U$ such that the following holds: for each $x\left(n_{2}\right) \in D\left(n_{2}\right) \cap \Sigma\left(n_{2}\right)$, exactly one of the following holds:

( $\alpha) x\left(n_{2}\right) \notin \Sigma_{1}\left(n_{2}\right)$, or

$\left(\beta^{\prime}\right) \bar{K}_{x\left(n_{2}\right)}=\left(u_{2, x\left(n_{2}\right)}^{b_{2, x\left(n_{2}\right)}} u_{3, x\left(n_{2}\right)}^{\left.b_{3, x\left(n_{2}\right)}^{\prime}\right)}\right)$ is a monomial (nonzero) ideal, where $b_{3, x\left(n_{2}\right)}^{\prime}=0$ if $m\left(x\left(n_{2}\right)\right)=2$. 
Once again, we assume w.l.o.g. that $n_{2}=0$ and define the following invariants from (16):

$$
A:=\max \left\{a_{Y}: Y \subseteq D \cap M_{2}\right\}, N:=\sharp\left\{Y \subseteq \Sigma: a_{Y}=A\right\} .
$$

Note that the case $A=0$, i.e. $R=\emptyset$ is dealt with by cases 1 ' and 3 . Assume now $A>0$ and we construct an $\left(\mathcal{E}, P_{\delta}\right)$-sequence $\pi(n): U(n) \rightarrow U$ such that $(A(n), N(n))<_{\operatorname{lex}}(A, N)$ with obvious notations, which will conclude the proof by descending induction on $(A, N)$.

Pick an irreducible component $Y \subseteq \Sigma$ with $a_{Y}=A$. If $Y$ is $\left(\mathcal{E}, P_{\delta}\right)$-permissible at all points, let $\pi(1): U(1) \rightarrow U$ be the blowing up along $Y$. There is an isomorphism $Y^{\prime}:=\pi(1)^{-1}(Y) \cap D(1) \simeq Y$ and we have $a_{Y^{\prime}}=a_{Y}-\mu$ ! if $Y^{\prime}$ meets $R(1)$. Therefore $(A(1), N(1))<_{\text {lex }}(A, N)$ in any case. There remains to reduce to this situation.

Assume now that $Y$ is not $\left(\mathcal{E}, P_{\delta}\right)$-permissible at closed points $x_{1}, \ldots, x_{r} \in Y$, $r \geq 1$. It can be assumed that $x_{j} \in R$ by the analysis in cases 1' and 3 above, $1 \leq j \leq r$. By $(18)$, we have $\nu_{x}\left(\delta, E_{x}\right)=0$ for $x \in Y \backslash\left\{x_{1}, \ldots, x_{r}\right\}, \nu_{x_{j}}\left(\delta, E_{x_{j}}\right)=1$ for $1 \leq j \leq r$.

Pick now $x \in\left\{x_{1}, \ldots, x_{r}\right\}$. We assume w.l.o.g. that $Y=V\left(u_{1}, u_{2}\right)$ locally at $x$. Comparing with $\left(\beta^{\prime}\right)$, we get a numerical criterion: $Y$ is not $\left(\mathcal{E}, P_{\delta}\right)$-permissible at $x$ if and only if:

$$
x \text { satisfies }\left(\beta^{\prime}\right) \text { with } m(x)=3 \text { and } b_{2, x}^{\prime}=0 .
$$

Case 4.2. $a_{3, x} / \mu !<1$. Let $\pi(1): U(1) \rightarrow U$ be the blowing up along $x, Y^{\prime}:=$ $D(1) \cap \pi(1)^{-1}(x), Y(1)$ be the strict transform of $Y$ and $\{x(1)\}:=Y^{\prime} \cap Y(1)$. We have $a_{Y^{\prime}}=a_{Y}+a_{3, x}-\mu !<A$ if $x(1) \in R(1)$. Therefore $(A(1), N(1)) \leq_{\operatorname{lex}}(A, N)$ in any case and we may substitute $(U, Y)$ by $(U(1), Y(1))$.

Case 4.3. $a_{3, x} / \mu ! \geq 1$. Let $Z \subset D \cap M_{2}$ be the Zariski closure of $V\left(u_{1}, u_{3}\right)$, so $Z \subset \Sigma$. Since $b_{3, x}^{\prime} \geq 1, Z$ is $\left(\mathcal{E}, P_{\delta}\right)$-permissible at all points and let $\pi(1): U(1) \rightarrow U$ be the blowing up along $Z, Z^{\prime}:=D(1) \cap \pi(1)^{-1}(Z), Y(1)$ be the strict transform of $Y$ and $\{x(1)\}:=Z^{\prime} \cap Y(1)$. We have $a_{3, x(1)}=a_{Z}-\mu$ ! $<A$. Therefore $(A(1), N(1)) \leq_{\text {lex }}(A, N)$ in any case and we may substitute as well $(U, Y)$ by $(U(1), Y(1))$.

We now analyze the structure of $\delta$ at $x(1)$ by (20) for both cases 4.2 and 4.3: if $b_{3, x}^{\prime}=1$, we have $\nu_{x(1)}\left(\delta, E(1)_{x(1)}\right)=0$, i.e. $Y(1)$ is $\left(\mathcal{E}, P_{\delta}\right)$-permissible at $x(1)$; if $b_{3, x}^{\prime} \geq 2$, we have $\nu_{x(1)}\left(\delta, E(1)_{x(1)}\right)=1$, and $x(1)$ again satisfies $(21)$ with $b_{3, x(1)}^{\prime}=$ $b_{3, x}^{\prime}-1$. Hence there exists an $\left(\mathcal{E}, P_{\delta}\right)$-sequence $\pi\left(b_{3, x}^{\prime}\right): U\left(b_{3, x}^{\prime}\right) \rightarrow U$ such that $\left(A\left(b_{3, x}^{\prime}\right), N\left(b_{3, x}^{\prime}\right)\right) \leq_{\text {lex }}(A, N)$ and the strict transform $Y\left(b_{3, x}^{\prime}\right)$ of $Y$ is $\left(\mathcal{E}, P_{\delta}\right)$-permissible at $\left\{x\left(b_{3, x}^{\prime}\right)\right\}:=\pi\left(b_{3, x}^{\prime}\right)^{-1}(x) \cap Y\left(b_{3, x}^{\prime}\right)$. Working in this way at each $x \in$ $\left\{x_{1}, \ldots, x_{r}\right\}$, this concludes the proof of lemma 4.6 .

We now prove proposition 4.2. We may assume w.l.o.g. that $n=0$ in lemma 4.6. To begin with, considerations similar to those in cases 1 and 1' in the previous proof show that it can be assumed that any point $x \in \Sigma$ with $\tau(x) \geq 2$ actually satisfies $\tau(x)=2$, and that there is a unique one-dimensional irreducible component $C_{x}$ of $\Sigma$ going through $x, C_{x}$ is $\left(\mathcal{E}, P_{\delta}\right)$-permissible at $x$. From now on, assume that $\tau(x)=1$.

We now apply lemma 4.4 and pick $x \in \pi(n)(\Sigma(n))$ with notations as in there. Since char $k=0$, there exists a maximal contact surface $D$ for the idealistic exponent $\mathcal{E}$ around $x$. Explicitly, pick $g \in \mathcal{I}_{x}$ with $\operatorname{ord}_{x} g=\mu$. Given a r.s.p. $\left(u_{1}, u_{2}, u_{3}\right)$ of 
$\mathcal{O}_{U, x}$ with $E_{x}=\operatorname{div}\left(u_{1} \cdots u_{e}\right), 1 \leq e=m(x) \leq 2$, we let

$$
z:=\frac{1}{(\mu-1) !} \frac{\partial^{\mu-1} g}{\partial u_{3}^{\mu-1}},
$$

and take $D:=\operatorname{div}(z)$. Then any $\left(\mathcal{E}, P_{\delta}\right)$-sequence above $x$ is centered on the strict transform of $D$. Without loss of generality, we add $u_{3}:=z$ to the convention $E_{x}=\operatorname{div}\left(u_{1} \cdots u_{e}\right)$. Note in particular that $Z_{x}=U_{3}$, with notations as in lemma 4.6.

The proof now runs parallel to that of lemma 4.6 and we only indicate those parts where the proof differs in what follows. If $x \in \Sigma$ and $g \in \mathcal{I}_{x}$, there is a unique expansion

$$
g=g_{0} u_{3}^{\mu}+\sum_{i=1}^{\mu} g_{i} u_{3}^{\mu-i},
$$

$g_{0} \in k\left[\left[u_{1}, u_{2}, u_{3}\right]\right] ; g_{i} \in k\left[\left[u_{1}, u_{2}\right]\right]$ and $\operatorname{ord}_{\left(u_{1}, u_{2}\right)} g_{i}>i$ for $1 \leq i \leq \mu$ and let

$$
c\left(\mathcal{I}_{x}\right):=\left(\left\{g_{i}^{\mu ! / i}: 1 \leq i \leq \mu, g \in \mathcal{I}_{x}\right\}\right) .
$$

It can be furthermore assumed w.l.o.g. that $c\left(\mathcal{I}_{x}\right)=\left(u_{1}^{a_{1, x}} u_{2}^{a_{2, x}}\right)$, with $a_{1, x}, a_{2, x} \in \mathbf{N}$, $\left(a_{1, x}+a_{2, x}\right) / \mu !>1$ for each $x \in R:=\{x \in \Sigma: \tau(x)=1\}$. Similarly, it can be assumed that $\Sigma$ has equal dimension one and $\Sigma \subseteq M:=M(0)$, where

$$
M(n):=D(n) \cap E(n) .
$$

With notations as in (19), let

$$
\bar{\delta}=\sum_{i=1}^{e} \bar{f}_{i, x} u_{i} \frac{\partial}{\partial u_{i}}+\sum_{j=e+1}^{2} \bar{f}_{j, x} \frac{\partial}{\partial u_{j}},
$$

where we still denote $u_{1}, u_{2}$ the images in $\mathcal{O}_{D, x}$ of the corresponding parameters of $\mathcal{O}_{U, x}$. Similarly, if $U(n) \rightarrow U$ is a finite composition of blowing ups at closed points and $x(n) \in D(n)$ is a closed point with r.s.p. $\left(u_{1, x(n)}, u_{2, x(n)}, u_{3, x(n)}\right), D(n)_{x(n)}=$ $\operatorname{div}\left(u_{3, x(n)}\right)$, we now let

$$
\bar{J}_{x(n)}:=\left(\bar{f}_{1, x(n)}, \bar{f}_{2, x(n)}\right), \bar{K}_{x(n)}:=\bar{J}_{x(n)}+\left(\bar{f}_{3, x(n)}\right) .
$$

For $x \in \Sigma_{1}$, let $\pi(1): U(1) \rightarrow U$ be the blowing up along $x$ and $x(1) \in \pi(1)^{-1}(x) \cap$ $\Sigma(1)$. We get the following transformation law from the blowing up formulæ [7] I.2.2.5:

$$
\bar{f}_{3, x(1)}=u_{j}^{-1-\epsilon} \bar{f}_{3, x}
$$

where $\left(u_{j}\right)$ is the ideal of $\pi(1)^{-1}(x)$ at $x(1), j=1$ or $j=2$, and $\epsilon=0$ (resp. $\epsilon=1$ ) if $\left(m(x)=1\right.$ and $\left.\operatorname{ord}_{x}\left(f_{2, x}, f_{3, x}\right)=1\right)$ or if $\left(m(x)=2\right.$ and $\left.\operatorname{ord}_{x} f_{3, x}=1\right)$ (resp. otherwise).

As in the proof of lemma 4.6, there exists a finite composition of blowing ups at closed points $U(n) \rightarrow U$ such that the following holds: for each $x(n) \in \Sigma(n) \cap D(n)$, exactly one of the following holds:

( $\alpha) x(n) \notin \Sigma_{1}(n)$, or

$\left(\beta^{\prime}\right) \bar{K}_{x(n)}=\left(u_{1, x(n)}^{b_{1, x(n)}^{\prime}} u_{2, x(n)}^{b_{2, x(n)}^{\prime}}\right)$ and $\left(\bar{f}_{3, x(n)}\right)=\left(u_{1, x(n)}^{c_{1, x(n)}} u_{2, x(n)}^{c_{2, x(n)}}\right)$ are monomial ideals, $1 \leq b_{1, x(n)}^{\prime}+b_{2, x(n)}^{\prime}<+\infty$, with $b_{2, x(n)}^{\prime}=c_{2, x(n)}=0$ if $m(x(n))=1$.

Moreover, $\left((\alpha)\right.$ or $\left.\left(\beta^{\prime}\right)\right)$ is preserved by further blowing ups at closed points. We take $n=0$ w.l.o.g. If $Y \subseteq M=M(0)$ is an irreducible curve meeting $R$, we let

$$
a_{Y}:=a_{i, x}, x \in Y \cap R \text { arbitrary with } \tau(x)=1, Y=V\left(u_{i}, u_{3}\right) \text { locally at } x .
$$


If $Y \subset D \cap M_{2}$ is an irreducible curve not meeting $R$, we simply let $a_{Y}:=0$. Define the following invariants:

$$
A:=\max \left\{a_{Y}: Y \subseteq \Sigma\right\}, N:=\sharp\left\{Y \subseteq \Sigma: a_{Y}=A\right\} .
$$

The case $A=0$, i.e. $\tau(x) \geq 2$ for each $x \in \Sigma$, is dealt with by blowing up each irreducible component of $\Sigma$ as in case 1' in the proof of lemma 4.6. Assume now that $A>0$. We construct an $\left(\mathcal{E}, P_{\delta}\right)$-sequence $\pi(n): U(n) \rightarrow U$ such that $(A(n), N(n))<_{\operatorname{lex}}(A, N)$ with obvious notations and thus conclude the proof by descending induction.

Pick an irreducible component $Y \subseteq \Sigma$ with $a_{Y}=A$. If $Y$ is $\left(\mathcal{E}, P_{\delta}\right)$-permissible at all points, we blow up $Y$ and get $(A(1), N(1))<_{\text {lex }}(A, N)$ as in the proof of lemma 4.6. We reduce below to this situation.

Let $x \in Y$ be such that $Y$ is not $\left(\mathcal{E}, P_{\delta}\right)$-permissible at $x$. We assume w.l.o.g. that $Y=V\left(u_{1}, u_{3}\right)$ locally at $x$. Using formulæ I.3.4.4.2 and I.3.4.4.3 of [7], our (nonnumerical) criterion is now: $Y$ is not $\left(\mathcal{E}, P_{\delta}\right)$-permissible at $x \in \Sigma$ if and only if:

$$
\left.x \in \Sigma_{1} \text { and (either } c_{1, x}=0 \text {, or }\left(f_{3, x} \in\left(u_{1}, u_{3}\right)^{2} \text { and } b_{1, x}^{\prime}=0\right)\right) \text {. }
$$

These conditions imply in particular that $m(x)=2$ and $b_{1, x}^{\prime}=0$ by $\left(\beta^{\prime}\right)$, so $b_{2, x}^{\prime} \geq 1$ since $x \in \Sigma_{1}$. Letting $Z \subset M$ to be the Zariski closure of $V\left(u_{2}, u_{3}\right)$, it follows that $Z$ is $\left(\mathcal{E}, P_{\delta}\right)$-permissible at all points provided $Z \subset \Sigma$.

We now consider two cases analogues of 4.2 (here $a_{2, x} / \mu$ ! $<1$ ) and 4.3 (here $a_{2, x} / \mu$ ! $\left.\geq 1\right)$ in the proof of lemma 4.6. Let $\pi(1): U(1) \rightarrow U$ be the blowing up along $x$ (resp. along $Z$ ) in the former case, i.e. $Z \not \subset \Sigma$ (resp. in the latter case, i.e. $Z \subset \Sigma)$. Let $Y(1)$ be the strict transform of $Y$ and $x(1):=\pi(1)^{-1}(x) \cap Y(1)$. By $(22)$ and (23), $Y(1)$ is $\left(\mathcal{E}, P_{\delta}\right)$-permissible at $x(1)$ if $\left(b_{1, x}^{\prime}, b_{2, x}^{\prime}\right)=(0,1)$; otherwise, $x$ (1) satisfies again one of the properties in (23) with $b_{2, x(1)}^{\prime} \leq b_{2, x}^{\prime}-1$. In any case $(A(1), N(1)) \leq_{\text {lex }}(A, N)$ and we may substitute $U, Y$ by $U(1), Y(1)$. Arguing by descending induction on $b_{2, x}^{\prime}$, we will get the strict transform $Y(n)$ to be $\left(\mathcal{E}, P_{\delta}\right)$ permissible at all points for some $n \geq 1$, together with $(A(n), N(n)) \leq_{\text {lex }}(A, N)$, which completes the proof.

4.2. Quadratic sequences along curves. We do not know if the following proposition remains valid for a regularity property which is not the refinement of the usual regularity property.

Proposition 4.7. Let $P$ be a regularity property which is a refinement of $P_{\text {reg }}$ and veryfies axioms 1,2,3 and 6 . Let $Y / k$ be a (regular) projective model of $K$ such that $\operatorname{RegP}(Y)=Y$. Then $P_{R S(Y)}$ verifies axiom 6 .

Proof. Let $X / k$ be a normal projective model of $K, x \in \operatorname{RegP}_{R S(Y)}(X)$ be such that $\operatorname{dim} \mathcal{O}_{X, x}=2$ and let $C:=\overline{\{x\}}$. Since $P$ verifies axioms 3 and 6 , it can be assumed w.l.o.g. that $C \subset \operatorname{RegP}(X)$. By proposition 3.2, it can be furthermore assumed that $C \subset \operatorname{RegP}_{Y}(X)$

Let $v^{\prime}$ be a fixed discrete $k$-valuation of $K$ dominating $\mathcal{O}_{X, C}$ with residue field a finite algebraic extension $k^{\prime}$ of $k(C)$, and $\bar{v}$ be any discrete valuation of $k^{\prime}$. We denote by $v$ the composed valuation $v=v^{\prime} \circ \bar{v}$, with group $\mathbf{Z}_{\text {lex }}^{2}$. Let

$$
X=: X_{0} \leftarrow X_{1} \leftarrow \cdots \leftarrow X_{n} \leftarrow \cdots
$$

be that sequence of blowing ups at closed points $z_{i} \in X_{i}$, where $z_{i}$ is the center of $v$. It is sufficient to prove that $\eta_{i}: X_{i} \rightarrow Y$ is a $P$-regular sequence at $z_{i}$ for some $i \geq 0$. 
Note that $\eta_{0}$ is defined at all points of $C$, since $C \subset \operatorname{RegP}_{Y}(X)$.

Assume that $\pi_{0}$ is not an isomorphism at $x$. We now apply definition 3.8 at the point $x \in X$. There exists a certain $Z_{0} \subset Y, Z_{0}$ either a closed point or an integral curve which is stably $P$-permissible at $\eta_{0}(x)$, the stalk $I$ of whose ideal at $\eta_{0}(x)$ is such that $I \mathcal{O}_{X, x}$ is principal.

If $\eta_{0}(x)$ is a closed point, then $I \mathcal{O}_{X_{i}, z_{i}}$ is principal for all $i>>0$ by proposition 3.2 applied to the blowing up of $Y$ along $Z_{0}$.

If $\eta_{0}(x)$ is the generic point of a curve, we necessarily have $Z_{0}=\overline{\left\{\eta_{0}(x)\right\}}$. Since $P$ verifies axiom 3 , there exists a composition of blowing ups at closed points $Y^{\prime} \rightarrow Y$ such that the strict transform $Z_{0}^{\prime}$ of $Z_{0}$ in $Y^{\prime}$ is stably $P$-permissible at all points. Let $M \subset \mathcal{O}_{Y, \eta_{0}\left(z_{0}\right)}$ be the maximal ideal. By proposition 3.2 applied to the blowing up of $Y$ along $\eta_{0}\left(z_{0}\right), M \mathcal{O}_{X_{i}, z_{i}}$ is principal for all $i>>$. Arguing by induction on the number of closed points blown up from $Y$ to $Y^{\prime}$, we may substitute $Y$ by $Y^{\prime}, X$ by $X_{n}$ for some $n>>0$, and thus assume that $Z_{0}$ is stably $P$-permissible at $\eta_{0}\left(z_{0}\right)$. Then it can be furthermore assumed that $\eta_{0}$ factors locally at $z_{0}$ through the blowing up along $Z_{0}$, applying once more proposition 3.2 to the blowing up of $Y$ along $Z_{0}$.

By induction on the number $n_{x}$ of blowing ups appearing in definition 3.8 w.r.t. $x$, we thus reduce w.l.o.g. to the case $n_{x}=0$, i.e. $\eta_{0}$ is an isomorphism at $x$.

As pointed out above, it can now be assumed that $\eta_{0}(C)$ is stably $P$-permissible at $\eta_{0}\left(z_{0}\right)$. In particular, $\eta_{0}(C)$ is regular at $\eta_{0}\left(z_{0}\right)$ and this implies that $\kappa\left(z_{i}\right)=\kappa\left(z_{0}\right)$ for all $i \geq 0$. Pick a r.s.p. $(u, v, w)$ of $\mathcal{O}_{Y, \eta_{0}\left(z_{0}\right)}$ in such a way that $(u, v)$ is the ideal of $C$ at $\eta_{0}\left(z_{0}\right)$. By standard arguments, there exists $i_{0} \geq 0$ such that the following holds for all $i \geq i_{0}$ :

(*) the strict transform of $\operatorname{div}(w)$ at $z_{i}$ is empty; the exceptional locus $E_{i}$ of $\eta_{i}$ at $z_{i}$ is irreducible, transverse to the strict transform of $C$ and maps to $\eta_{0}\left(z_{0}\right)$.

Pick $i \geq i_{0}$ and let $w_{i} \in \mathcal{O}_{X_{i}, z_{i}}$ be a local equation of $E_{i}$. By construction, $\mathcal{O}_{X_{i}, z_{i}}$ has a r.s.p. $\left(u_{i}, v_{i}, w_{i}\right)$ where $w=\gamma_{i} w_{i}^{c_{i}}$ for some $c_{i}>0$ and $\gamma_{i} \in \mathcal{O}_{X_{i}, z_{i}}$ invertible; $u_{i}=u / w_{i}^{a_{i}}, v_{i}=v / w_{i}^{b_{i}}$ for some $a_{i}, b_{i} \geq 0$. We define a discrete $k$-valuation of $K$ as follows: given a nonzero series in $\widehat{\mathcal{O}_{X_{i}, z_{i}}} \simeq \kappa\left(z_{0}\right)\left[\left[u_{i}, v_{i}, w_{i}\right]\right]$, we let

$$
\hat{\nu}\left(\sum_{(\alpha, \beta, \gamma) \in \mathbf{N}^{3}} \lambda_{\alpha \beta \gamma} u_{i}^{\alpha} v_{i}^{\beta} w_{i}^{\gamma}\right):=\min \left\{\alpha+\beta+\gamma: \lambda_{\alpha \beta \gamma} \neq 0\right\} \in \mathbf{N} .
$$

Then $\hat{\nu}$ extends by linearity to a discrete $k$-valuation of $Q F\left(\widehat{\mathcal{O}_{X_{i}, z_{i}}}\right)$ and we let $\nu$ be the restriction of $\hat{\nu}$ to $K$.

Now, the value group $\mathbf{Z}$ of $\nu$ is generated by the values $\nu(u)=1+a_{i}, \nu(v)=$ $1+b_{i}, \nu(w)=c_{i}$, so we have

$$
\text { g.c.d }\left(1+a_{i}, 1+b_{i}, c_{i}\right)=1 \text {. }
$$

On the other hand, the residue field $\kappa(\nu)$ of $\nu$ is equal to

$$
\kappa\left(z_{0}\right)\left(\operatorname{cl}\left(u_{i} / w_{i}\right), \operatorname{cl}\left(v_{i} / w_{i}\right)\right),
$$

where cl denotes the residue map of the valuation ring $R_{\nu}$ of $\nu$. Doing similar computations in $\mathcal{O}_{Y, \eta\left(z_{0}\right)}$, we get

$$
\kappa(\nu)=\kappa\left(z_{0}\right)\left(\left\{\operatorname{cl}\left(u^{\alpha} v^{\beta} w^{\gamma}\right):\left(1+a_{i}\right) \alpha+\left(1+b_{i}\right) \beta+c_{i} \gamma=0\right\}\right) .
$$


Let $\mathbf{v}:=\left(1+a_{i}, 1+b_{i}, c_{i}\right) \in\left(\mathbf{Z}^{3}\right)^{\vee}$. By $(24)$, the map $\mathbf{Z}^{3} \longrightarrow \mathbf{v} \longrightarrow \mathbf{Z}$ induced by $\mathbf{v}$ is surjective; by (25) and (26), its kernel $K \simeq \mathbf{Z}^{2}$ projects isomorphically to the first two coordinates lattice $\mathbf{Z}^{2} \subset \mathbf{Z}^{3}$. This implies that $c_{i}=1$, and we will consequently take $\gamma_{i}=1$ in what follows.

After possibly switching $u$ and $v$, it can be assumed that $b_{i} \geq a_{i}$. If equality holds, $\eta_{i}$ obviously factors as a composition of point blowing ups at $z_{i}$ and the proposition is proved in this case.

Assume now that $d_{i}:=b_{i}-a_{i}>0$ and note that $d_{i}$ is independent of $i \geq i_{0}$ since $\left(^{*}\right)$ above holds. Let $Z \subset Y$ be the Zariski closure of the germ of curve $V(v, w)$. Once more, it can be assumed that $Z$ is stably $P$-permissible at $\eta_{0}\left(z_{0}\right)$ while preserving condition $(*)$ for all $i \geq i_{1}$ for some $i_{1} \geq i_{0}$. Substituting $Y$ by its blowing up along $Z$ preserves these assumptions and changes $\left(a_{i}, b_{i}\right)$ to $\left(a_{i}, b_{i}-1\right)$, hence $d_{i}$ to $d_{i}-1$. Iterating $d_{i}$ times this step reduces to the situation of the previous paragraph, thus completes the proof.

4.3. Applications. Applying our theorem 2.5, F. Cano, C. Roche and M. Spivakovsky obtain corollary 4.9 below (case $P=P_{\text {reg }}$ of the next proposition) from their local uniformization theorem for vector fields (see part 3 of [10]). A slight refinement of their argument yields the following:

Proposition 4.8. Assume that $k$ is algebraically closed and chark $=0$. Let $X / k$ be a regular projective model of $K$ and $\delta \in \operatorname{Der}_{K / k}$ be a nonzero element. Let $P$ be a regularity property which satisfies the assumptions of proposition 4.1 and verifies axioms 5 and 6.

There exists a projective model $Y / k$ of $K$ such that $\operatorname{RegP}_{\delta}(Y)=Y$, together with a birational projective morphism $\pi: Y \rightarrow X$ such that $\pi$ is an isomorphism above $\operatorname{RegP}_{\delta}(X)$.

Proof. This proposition will be a consequence of theorem 2.5 applied to the property $P_{\delta}$ and to the model $X$ once we have proved that $P_{\delta}$ verifies axioms 1 to 6 .

Since $P$ verifies axioms $1,2,3$ and 6 , so does $P_{\delta}$ by proposition 3.7. By proposition $4.2, P_{\delta}$ verifies axiom 4 .

The hard part is to prove that $P_{\delta}$ verifies axiom 5 (local uniformization). This is because, for a given $k$-valuation ring $V / k$ of $K$, the local uniformization $(M, Y)$ produced by theorem 1 of [10] is obtained from any given model $M_{0} / k$ of $K$ which is regular (in the usual sense) at the center of $V$ by blowing up centers which are locally regular at the center of $V$. Since $P$ verifies axiom 5 , it can be assumed that $M_{0}$ is $P$-regular at the center of $V$ to begin with. Since $P$ satisfies the assumptions of proposition 4.1, $M$ will consequently be $P_{\delta}$-regular at the center $Y$ of $V$.

Since the usual regularity property satisfies the assumptions of the proposition (case $\mu=1$ of proposition 3.5 ), we get:

Corollary 4.9. [10] (Cano, Roche, Spivakovsky). Assume that $k$ is algebraically closed and chark $=0$. Let $X / k$ be a regular projective model of $K$ and $\delta \in \operatorname{Der}_{K / k}$ be a nonzero element.

There exists a projective model $Y / k$ of $K$ such that $\delta$ is log-elementary at all points $y \in Y$, together with a birational projective morphism $\pi: Y \rightarrow X$. Moreover, $\pi$ is an isomorphism above each $x \in X$ such that $\delta$ is log-elementary at $x$. 
Theorem 2.5 can also be used to prove the following local form of Hironaka's Strong Factorization Conjecture [18], since Local Uniformization has been proved in this context [14] [23]. Note that we get an optimal control on the fundamental loci.

Theorem 4.10. Assume that chark $=0$. Let $\pi: Y_{2} \rightarrow Y_{1}$ be a birational (projective) morphism of regular projective models of $K$.

There exists a regular projective model $Y / k$ of $K$, together with birational projective morphism $\pi_{i}: Y \rightarrow Y_{i}, i=1,2$, such that $\pi_{i}$ is locally a monoidal sequence at each $y \in Y$ and for both $i=1,2$ and the following holds:

(i) the fundamental locus $\mathcal{F}_{2}$ of $\pi_{2}^{-1}$ is given by

$$
\mathcal{F}_{2}:=\left\{y \in Y_{2}: \pi \text { is not a monoidal sequence at } y\right\} .
$$

(ii) $\pi\left(\mathcal{F}_{2}\right)$ is a finite set contained in the fundamental locus $\mathcal{F}$ of $\pi$. In particular, the fundamental locus $\mathcal{F}_{1}$ of $\pi_{1}^{-1}$ is equal to $F$.

Proof. Consider the regularity properties $P_{i}:=P_{\mathrm{reg}, R S\left(Y_{i}\right)}, i=1,2$ from definition 3.9. Let $P:=P_{1} \wedge P_{2}$ be the conjunction of $P_{1}$ and $P_{2}$. We claim that $Y:=X^{\prime}$ satisfies the conclusion of the theorem if and only if $X^{\prime}$ satisfies the conclusion of theorem 2.5 w.r.t. $P$ and for $X:=Y_{2}$.

Clearly $\pi_{i}$ is a monoidal sequence at each $y \in Y$ and for both $i=1,2$ if and only if $\operatorname{RegP}(Y)=Y$. By definition of $P$, we have $\operatorname{SingP}\left(Y_{2}\right)=\mathcal{F}_{2}$, so (i) is a mere reformulation of the content of theorem 2.5. We now prove that (ii) is a consequence of (i). Let $Z \subset \pi^{-1}(\mathcal{F})$ be an integral curve with generic point $x$ such that $\pi(Z)$ is a curve. Then

$$
\mathcal{O}_{Y_{1}, \pi(x)}<\mathcal{O}_{Y_{2}, x}
$$

is an iterated quadratic transform by Abhyankar's theorem 3 [2], i.e. $x \in \operatorname{RegP}\left(Y_{2}\right)$ and the conclusion easily follows.

There hence remains to prove that $P$ verifies axioms 1 to 6 . Since the usual regularity property $P_{\text {reg }}$ verifies axioms $1,2,3$ and 6 by proposition 3.5 with $\mu=1$, so does $P$ by propositions $3.10,4.7$ and 3.11 . By proposition $4.1, P$ verifies axiom 4 . Finally, that $P$ verifies axiom 5 is the content of theorem 0.1 [23] (this is where the characteristic zero assumption is used).

The following example was brought to our attention by S.D. Cutkosky. It shows that not every birational morphism of regular projective threefolds which is everywhere a monoidal sequence is globally a composition of blowing ups with regular centers.

Example 4.11. [27] Take $P=P_{\text {reg }}$ the usual regularity property. Let $\pi: Y \rightarrow X:=$ $\mathbf{P}_{k}^{3}$ be the toric projective morphism corresponding to the following regular subdivision of $\mathbf{R}_{\geq 0}^{3}$ at the origin $x=(1: 0: 0: 0)$ :

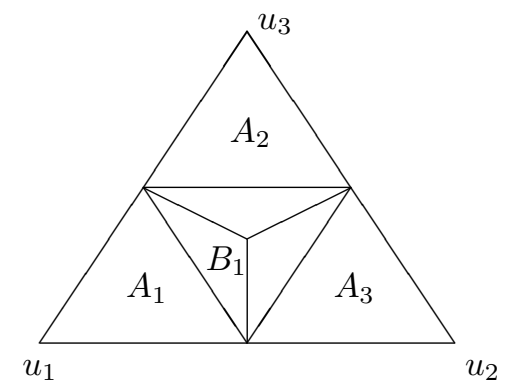


The regular variety $Y$ is covered by six charts above the origin. To prove that $\pi$ is everywhere locally a monoidal sequence, i.e. that $\operatorname{Reg}_{R S(X)}(Y)=Y$, it is sufficient by symmetry to check it on each of the charts $A_{1}$ and $B_{1}$ in the above figure.

Chart $A_{1}$ corresponds to the inclusion $k\left[u_{1}, u_{2}, u_{3}\right] \subset A_{1}:=k\left[u_{1} / u_{2} u_{3}, u_{2}, u_{3}\right]$ of affine algebras, which factors as a monoidal sequence as follows:

$$
k\left[u_{1}, u_{2}, u_{3}\right] \subset k\left[u_{1} / u_{2}, u_{2}, u_{3}\right] \subset A_{1} .
$$

Chart $B_{1}$ corresponds to the inclusion $k\left[u_{1}, u_{2}, u_{3}\right] \subset B_{1}:=k\left[u_{1} / u_{2}, u_{1} / u_{3}, u_{2} u_{3} / u_{1}\right]$ of affine algebras, which factors as a monoidal sequence as follows:

$$
k\left[u_{1}, u_{2}, u_{3}\right] \subset k\left[u_{1} / u_{2}, u_{2}, u_{3}\right] \subset k\left[u_{1} / u_{2}, u_{2}, u_{2} u_{3} / u_{1}\right] \subset B_{1} .
$$

On the other hand, $\pi$ is an isomorphism away from the union of all three coordinate axes $l_{1}: x_{2}=x_{3}=0, l_{2}: x_{1}=x_{3}=0, l_{3}: x_{1}=x_{2}=0$ going through $x$. But $\pi$ does not factor through the blowing up at $x$, nor through the blowing up of any of the lines $l_{1}, l_{2}$ or $l_{3}$. This can be easily checked: for example, none of the corresponding ideals to these closed subsets is principal on all three charts $A_{1}, A_{2}$ and $A_{3}$. Therefore $\pi$ is not a composition of blowing ups with regular centers.

\section{Constructing P-Regular models: proof of theorem 2.4.}

From now on, $P$ is a regularity property of local rings $R \subseteq K$, essentially of finite type over $k$ and such that $Q F(R)=K$, verifying axioms 1 to 5 . Let $X / k$ be a projective model of $K$. In order to prove theorem 2.4, the main point is to prove the following:

Proposition 5.1. Let $X_{1} / k$ and $X_{2} / k$ be two projective models of $K$. There exists a normal and projective model $Y / k$ of $K$, together with morphisms $\pi_{i}: Y \rightarrow X_{i}$, such that $\pi_{i}^{-1}\left(\operatorname{RegP}\left(X_{i}\right)\right) \subseteq \operatorname{Reg} \mathrm{P}(Y)$ for $i=1,2$.

Proof. The proof is long and essentially an adaptation of Zariski's proof of his Patching Theorem ([33], Fundamental Theorem on p. 539). We split it into various steps.

Step 1. It can be assumed that $X_{1}$ and $X_{2}$ are normal by (i) of axiom 2 .

Step 2. It can be assumed that the rational map $\eta: X_{2} \cdots \rightarrow X_{1}$ is a morphism. Namely, let $Z$ be the normalization of the closure of the graph of $\eta$, so $Z$ dominates both $X_{1}$ and $X_{2}$. Note that $Z \rightarrow X_{2}$ is projective, hence the blowing up along a certain ideal sheaf $\mathcal{I} \subseteq \mathcal{O}_{X_{2}}$.

By (i)' and (ii) of axiom 4 applied to the pair $\left(X_{2}, \mathcal{I}\right)$, there exists a projective birational morphism $\pi: Z_{1} \rightarrow X_{2}$ such that $\mathcal{I O}_{Z_{1}}$ is locally principal (hence $\pi$ factorize through $Z$ ) and $\pi^{-1}\left(\operatorname{RegP}\left(X_{2}\right)\right) \subseteq \operatorname{RegP}\left(Z_{1}\right)$. Therefore we may substitute $X_{2}$ by the normalization of $Z_{1}$, applying once again (i) of axiom 2 .

Step 3. Given a birational morphism $\eta: X_{2} \rightarrow X_{1}$ of normal projective varieties, we denote by $\mathcal{F}_{\eta} \subset X_{1}$ the fundamental locus of $\eta^{-1}$. Since $X_{1}$ is normal, $\mathcal{F}_{\eta}$ has codimension at least two, so it contains finitely many distinct irreducible curves. We need the following definition.

Definition 5.2. With notations as above, let $x \in \operatorname{RegP}\left(X_{1}\right)$ be such that $\operatorname{dim} \mathcal{O}_{X_{1}, x}=$ 2 and let $Z_{x}:=\overline{\{x\}}$. We say that $\eta$ is factorizable above $x$ if the localization map of $\eta$ at $x$

$$
\eta_{x}: X_{2, x}:=X_{2} \times \operatorname{Spec} \mathcal{O}_{X_{1}, x} \rightarrow \operatorname{Spec} \mathcal{O}_{X_{1}, x}
$$


is a composition of normalized blowing ups of (closed) points. In this case, we let $n_{x} \geq 0$ be the number of such normalized blowing ups of points.

We say that $\eta$ is factorizable if for each $x \in \operatorname{RegP}\left(X_{1}\right)$ such that $\operatorname{dim} \mathcal{O}_{X_{1}, x}=2, \eta$ is factorizable at $x$.

Note that for $x$ as in the definition such that $\eta$ is factorizable above $x$, there is an equivalence

$$
n_{x}>0 \Leftrightarrow Z_{x} \subseteq \mathcal{F}_{\eta} .
$$

By the following lemma, it can be assumed that $\eta: X_{2} \rightarrow X_{1}$ is factorizable, since $\mathcal{F}_{\eta}$ contains finitely many distinct irreducible curves.

Lemma 5.3. Let $\eta: X_{2} \rightarrow X_{1}$ be a birational morphism of normal projective models of $K$ and $x \in \operatorname{RegP}\left(X_{1}\right)$ be such that $\operatorname{dim} \mathcal{O}_{X_{1}, x}=2$. There exists a projective birational morphism $\pi^{\prime}: X_{2}^{\prime} \rightarrow X_{2}, X_{2}^{\prime}$ normal such that

(i) $\mathcal{F}_{\eta^{\prime}}=\mathcal{F}_{\eta}$, where $\eta^{\prime}: X_{2}^{\prime} \rightarrow X_{1}$ is the composed map;

(ii) $\pi^{\prime-1}\left(\operatorname{RegP}\left(X_{2}\right)\right) \subseteq \operatorname{RegP}\left(X_{2}^{\prime}\right)$;

(iii) $\eta^{\prime}$ is factorizable above $x$.

Proof. By Zariski's theorem on resolution of surface singularities by successive normalized blowing ups (p.688 [30]) and theorem 3 [2], there exists a composition of, say $n_{x} \geq 0$, normalized blowing ups of points $Z_{x} \rightarrow X_{2, x}$ such that the composed map $Z_{x} \rightarrow \operatorname{SpecO}_{X_{1}, x}$ is a composition of normalized blowing ups of points. The lemma holds with $X_{2}^{\prime}=X_{2}$ if $n_{x}=0$.

Assume that $n_{x} \geq 1$ and let $X_{2, x}^{\prime} \rightarrow X_{2, x}$ be the first of these normalized blowing ups, centered at $x^{\prime} \in X_{2, x}$. Let $Z_{x^{\prime}}$ be the Zariski closure of $x^{\prime}$ in $X_{2}$, so $Z_{x^{\prime}}$ is a curve mapping surjectively to $Z_{x}$; we consider two cases:

Case 1: $x^{\prime} \notin \operatorname{RegP}\left(X_{2}\right)$. Then $Z_{x^{\prime}} \cap \operatorname{RegP}\left(X_{2}\right)=\emptyset$, since $\operatorname{RegP}\left(X_{2}\right)$ is Zariski open (axiom 1). Let $\pi^{\prime}: X_{2}^{\prime} \rightarrow X_{2}$ be the normalized blowing up along $Z_{x^{\prime}}$ and $\eta^{\prime}: X_{2}^{\prime} \rightarrow$ $X_{1}$ be the composed map. We have $\mathcal{F}_{\eta^{\prime}}=\mathcal{F}_{\eta}$ and $\pi^{\prime-1}\left(\operatorname{RegP}\left(X_{2}\right)\right) \subseteq \operatorname{RegP}\left(X_{2}^{\prime}\right)$. On the other hand, $X_{2, x}^{\prime}=X_{2}^{\prime} \times \operatorname{Spec} \mathcal{O}_{X_{1}, x}$, so replacing $X_{2}$ by $X_{2}^{\prime}$ yields a reduction in $n_{x}$.

Case 2: $x^{\prime} \in \operatorname{RegP}\left(X_{2}\right)$. We use axiom 3 to $x^{\prime}$. There exists a composition $X_{2,0} \rightarrow X_{2}$ of normalized blowing ups of closed points belonging to the successive strict transforms of $Z_{x^{\prime}}$ such that the strict transform $Z_{0}^{\prime}$ of $Z_{x^{\prime}}$ in $X_{2,0}$ is (stably) $P$-permissible at all points. Let $X_{2}^{\prime} \rightarrow X_{2,0}$ be the normalized blowing up along $Z_{0}^{\prime}$, $\pi^{\prime}: X_{2}^{\prime} \rightarrow X_{2}$ and $\eta^{\prime}: X_{2}^{\prime} \rightarrow X_{1}$ be the composed map. We have $\mathcal{F}_{\eta^{\prime}}=\mathcal{F}_{\eta}$ and $\pi^{\prime-1}\left(\operatorname{RegP}\left(X_{2}\right)\right) \subseteq \operatorname{RegP}\left(X_{2}^{\prime}\right)$ by axiom 2 and definition 2.2. On the other hand, $X_{2, x}^{\prime}=X_{2}^{\prime} \times \operatorname{Spec} \mathcal{O}_{X_{1}, x}$, so replacing $X_{2}$ by $X_{2}^{\prime}$ also yields a reduction in $n_{x}$.

Note that if $\eta: X_{2} \rightarrow X_{1}$ is factorizable, an immediate consequence of axiom 2 is the following:

$$
\operatorname{RegP}\left(X_{1}\right) \cap \eta\left(\operatorname{SingP}\left(X_{2}\right)\right) \text { is a finite set. }
$$

Step 4. The following definition is essential. This step consists in removing bad points for $\operatorname{SingP}\left(X_{1}\right)$.

Definition 5.4. Let $\eta: X_{2} \rightarrow X_{1}$ be a birational morphism of normal projective models of $K$ and $x \in \operatorname{RegP}\left(X_{1}\right)$ be such that $\operatorname{dim} \mathcal{O}_{X_{1}, x}=2$. Let $\Sigma_{1}:=\operatorname{SingP}\left(X_{1}\right)$. We say that a point $x \in \operatorname{RegP}\left(X_{1}\right)$ such that $\operatorname{dim} \mathcal{O}_{X_{1}, x}=2$ is bad for $\Sigma_{1}$ if $Z_{x}:=$ $\overline{\{x\}} \subseteq \mathcal{F}_{\eta}$ and $Z_{x}$ meets $\Sigma_{1}$. 
Assume that $\eta: X_{2} \rightarrow X_{1}$ is factorizable above each $x \in \operatorname{RegP}\left(X_{1}\right), \operatorname{dim} \mathcal{O}_{X_{1}, x}=2$, which is bad for $\Sigma_{1}$. We now define our main invariant, with notations as in definition 5.2 : let $N_{\eta}:=\sum_{x} n_{x} \geq 0$, where the sum runs over all $x \in \operatorname{RegP}\left(X_{1}\right), \operatorname{dim} \mathcal{O}_{X_{1}, x}=2$ which are bad for $\Sigma_{1}$. Let

$$
\Phi:=\Sigma_{1} \cup \Sigma_{b} \cup \eta\left(\operatorname{SingP}\left(X_{2}\right)\right),
$$

where

$$
\Sigma_{b}:=\bigcup_{x \text { bad }} Z_{x} .
$$

Consider now a commutative diagram of birational projective morphisms

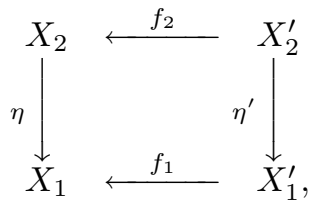

$X_{1}^{\prime}$ and $X_{2}^{\prime}$ normal, such that

(i) $f_{i}^{-1}\left(\operatorname{RegP}\left(X_{i}\right)\right) \subseteq \operatorname{RegP}\left(X_{i}^{\prime}\right), i=1,2$, and

(ii) $f_{1}$ induces an isomorphism $X_{1}^{\prime} \backslash f_{1}^{-1}(\Phi) \simeq X_{1} \backslash \Phi$.

We let $\Sigma_{1}^{\prime}:=f_{1}^{-1}\left(\Sigma_{1}\right)$ and extend the definition of bad points as follows:

Definition 5.5. With notations as above, let $x^{\prime} \in f_{1}^{-1}\left(\operatorname{Reg} \mathrm{P}\left(X_{1}\right)\right)$ be such that $\operatorname{dim} \mathcal{O}_{X_{1}^{\prime}, x^{\prime}}=2$. We say that $x^{\prime}$ is bad for $\Sigma_{1}^{\prime}$ if $Z_{x^{\prime}}:=\overline{\left\{x^{\prime}\right\}} \subseteq \mathcal{F}_{\eta^{\prime}}$ and $Z_{x^{\prime}}$ meets $\Sigma_{1}^{\prime}$.

If moreover $\eta^{\prime}$ is factorizable above each point $x^{\prime} \in f_{1}^{-1}\left(\operatorname{RegP}\left(X_{1}\right)\right)$ which is bad for $\Sigma_{1}^{\prime}$, we let $N_{\eta^{\prime}}:=\sum_{x^{\prime}} n_{x^{\prime}} \geq 0$, where the sum runs over all $x^{\prime} \in f_{1}^{-1}\left(\operatorname{RegP}\left(X_{1}\right)\right)$, $\operatorname{dim} \mathcal{O}_{X_{1}^{\prime}, x^{\prime}}=2$ which are bad for $\Sigma_{1}^{\prime}$.

Lemma 5.6. Assume that $\eta: X_{2} \rightarrow X_{1}$ is factorizable above each $x \in \operatorname{RegP}\left(X_{1}\right)$, $\operatorname{dim} \mathcal{O}_{X_{1}, x}=2$, which is bad for $\Sigma_{1}$. There exists a diagram (29), satisfying properties (i) and (ii) such that there exists no $x^{\prime} \in f_{1}^{-1}\left(\operatorname{RegP}\left(X_{1}\right)\right)$, $\operatorname{dim} \mathcal{O}_{X_{1}^{\prime}, x^{\prime}}=2$, which is bad for $\Sigma_{1}^{\prime}$.

Proof. Assume that $N_{\eta} \geq 1$ and let $x \in \operatorname{RegP}\left(X_{1}\right), \operatorname{dim} \mathcal{O}_{X_{1}, x}=2$ be bad for $\Sigma_{1}$. By axiom 3 , there exists a composition of normalized blowing ups

$$
X_{1}=: X_{1,0} \leftarrow \cdots \leftarrow X_{1, m}=: X_{1}^{\prime}
$$

at closed points $z_{i} \in \operatorname{RegP}\left(X_{1, i}\right), 1 \leq i \leq m-1$, such that the strict transform $Z_{x}^{\prime}$ of $Z_{x}$ in $X_{1}^{\prime}$ is (stably) $P$-permissible at all points in $Z_{x}^{\prime} \cap \operatorname{RegP}\left(X_{1}^{\prime}\right)$. By axiom 4 applied to the pair $\left(X_{2}, M_{z_{1}}\right)$, there exists a projective birational morphism $f_{2,1}: X_{2,1} \rightarrow X_{2}$ such that $M_{p_{1}} \mathcal{O}_{X_{2,1}}$ is locally principal (hence $X_{2,1} \rightarrow X_{1}$ factorize through $X_{1,1}$ ) and $f_{2,1}^{-1}\left(\operatorname{RegP}\left(X_{2}\right)\right) \subseteq \operatorname{RegP}\left(X_{2,1}\right)$. By (i) of axiom 2 , it can be furthermore assumed that $X_{2,1}$ is normal. By (iii) of axiom 4 and (27), $f_{2,1}$ is an isomorphism above $\eta^{-1}\left(X_{1} \backslash \Phi\right)$ and above each $x \in \operatorname{RegP}\left(X_{1}\right)$ which is bad for $\Sigma_{1}$.

By induction on $i, 1 \leq i \leq m$, we construct a sequence of birational projective morphisms

$$
X_{2}=: X_{2,0} \leftarrow \cdots \leftarrow X_{2, m}=: X_{2}^{\prime},
$$

with $X_{2}^{\prime}$ normal, such that $X_{2}^{\prime} \rightarrow X_{1}$ factorizes through $X_{1}^{\prime}$. Let $f_{i}: X_{i}^{\prime} \rightarrow X_{i}, i=1,2$ and $\eta^{\prime}: X_{2}^{\prime} \rightarrow X_{1}^{\prime}$. We have thus constructed a diagram (29) with the above properties (i) and (ii) and get defined $\Sigma_{1}^{\prime}:=f_{1}^{-1}\left(\Sigma_{1}\right)$. Moreover, $f_{2}$ is an isomorphism above each $x \in \operatorname{RegP}\left(X_{1}\right)$ which is bad for $\Sigma_{1}$. Therefore $f_{1}$ induces a one-to-one correspondence between bad points for $\Sigma_{1}^{\prime}$ and bad points for $\Sigma_{1}$ and $f_{1}$ is an isomorphism at 
$x^{\prime}$ whenever $x^{\prime}$ is bad for $\Sigma_{1}^{\prime}$. This proves that $\eta_{x^{\prime}}^{\prime}=\eta_{x}$ whenever $x^{\prime}$ is bad for $\Sigma_{1}^{\prime}$ and in particular $N_{\eta^{\prime}}=N_{\eta}$. Arguing by induction on the number of bad $x$ 's for $\Sigma_{1}$, we get a diagram (29) satisfying (i) and (ii), $N_{\eta^{\prime}}=N_{\eta}$, and such that the following holds:

(iii) $Z_{x^{\prime}}$ is (stably) $P$-permissible at all points in $Z_{x^{\prime}} \cap \operatorname{RegP}\left(X_{1}^{\prime}\right)$ and for each $x^{\prime} \in \operatorname{RegP}\left(X_{1}^{\prime}\right), \operatorname{dim} \mathcal{O}_{X_{1}^{\prime}, x^{\prime}}=2$, which is bad for $\Sigma_{1}^{\prime}$.

Let $x^{\prime}$ be bad for $\Sigma_{1}^{\prime}$ and $\mathcal{I}_{x^{\prime}} \subset \mathcal{O}_{X_{1}^{\prime}}$ be the ideal of $Z_{x^{\prime}}$. Since $\eta^{\prime}$ is factorizable above $x^{\prime}$, the complement $C_{x^{\prime}}$ of the Zariski open set

$$
U_{x^{\prime}}:=\left\{z^{\prime} \in X_{2}^{\prime} \mid \mathcal{I}_{x^{\prime}} \mathcal{O}_{X_{2}^{\prime}} \text { is principal }\right\}
$$

satisfies $\eta^{\prime}\left(C_{x^{\prime}}\right) \subset Z_{x^{\prime}}$ and $\eta^{\prime}\left(C_{x^{\prime}}\right)$ is a finite set.

By axiom 4 applied to the pair $\left(X_{2}^{\prime}, \mathcal{I}_{x^{\prime}} \mathcal{O}_{X_{2}^{\prime}}\right)$ and (i) of axiom 2 , there exists a birational projective morphism $f_{2}^{\prime}: X_{2}^{\prime \prime} \rightarrow X_{2}^{\prime}, X_{2}^{\prime \prime}$ normal, such that

$$
f_{2}^{\prime-1}\left(\operatorname{Reg} \mathrm{P}\left(X_{2}^{\prime}\right)\right) \subseteq \operatorname{RegP}\left(X_{2}^{\prime \prime}\right),
$$

the ideal $\mathcal{I}_{x^{\prime}} \mathcal{O}_{X_{2}^{\prime \prime}}$ is locally principal and $f_{2}^{\prime}$ is an isomorphism above $U_{x^{\prime}} \cap \operatorname{RegP}\left(X_{2}^{\prime}\right)$. Let $\eta^{\prime \prime}: X_{2}^{\prime \prime} \rightarrow X_{1}^{\prime}$ be the composed map. By construction,

$$
\mathcal{F}_{\eta^{\prime \prime}} \cap f_{1}^{-1}\left(\operatorname{RegP}\left(X_{1}\right)\right)=\mathcal{F}_{\eta^{\prime}} \cap f_{1}^{-1}\left(\operatorname{RegP}\left(X_{1}\right)\right)
$$

and we have $\eta_{x^{\prime}}^{\prime \prime}=\eta_{x^{\prime}}^{\prime}$ for each bad $x^{\prime}$ for $\Sigma_{1}^{\prime}$. Replacing $X_{2}^{\prime}$ by $X_{2}^{\prime \prime}$ and arguing by induction on the number of bad $x^{\prime}$ 's for $\Sigma_{1}^{\prime}$, we achieve a diagram (29) satisfying the above properties (i), (ii) and (iii), $N_{\eta^{\prime}}=N_{\eta}$, and such that the following holds:

(iv) $\mathcal{I}_{x^{\prime}} \mathcal{O}_{X_{2}^{\prime}}$ is locally principal for each bad $x^{\prime}$ for $\Sigma_{1}^{\prime}$.

Let $x^{\prime}$ be bad for $\Sigma_{1}^{\prime}$. By (iv), $\eta^{\prime}$ then factors through the normalized blowing up $f_{1}^{\prime}$ : $X_{1}^{\prime \prime} \rightarrow X_{1}^{\prime}$ along $Z_{x^{\prime}}$. By axiom 2 and (iii), we have $f_{1}^{\prime-1}\left(\operatorname{RegP}\left(X_{1}^{\prime}\right)\right) \subseteq \operatorname{RegP}\left(X_{1}^{\prime \prime}\right)$. Replacing $X_{1}^{\prime}$ by $X_{1}^{\prime \prime}$ therefore gives a new diagram (29) with $N_{\eta^{\prime}}=N_{\eta}-1$. Arguing by induction on $N_{\eta}$, we eventually get a diagram (29) having property (i) and (ii), and such that $N_{\eta^{\prime}}=0$.

Step 5. Pick a diagram (29) satisfying the conclusion of lemma 5.6. Since there is no bad $x^{\prime}$ for $\Sigma_{1}^{\prime}$, any irreducible component of $\mathcal{F}_{\eta^{\prime}}$ is either identically contained in $f_{1}^{-1}\left(\operatorname{RegP}\left(X_{1}\right)\right)$ or is contained in $\Sigma_{1}^{\prime}$. Let $\mathcal{F}_{1}:=\mathcal{F}_{\eta^{\prime}} \cap f_{1}^{-1}\left(\operatorname{RegP}\left(X_{1}\right)\right), U_{1}:=X_{1}^{\prime} \backslash \mathcal{F}_{1}$ and $U_{2}:=f_{1}^{-1}\left(\operatorname{RegP}\left(X_{1}\right)\right)$. We have $X_{1}^{\prime}=U_{1} \cup U_{2}$. Let $U:=U_{1} \cap U_{2}$. By (i) and definition of $\mathcal{F}_{1}$, we have

$$
U \subseteq \operatorname{RegP}\left(X_{1}^{\prime}\right) \text { and } \eta^{\prime-1}(U) \simeq U .
$$

Since $\eta^{\prime}$ is birational and projective, it is the blowing up along a certain ideal sheaf $\mathcal{I} \subseteq \mathcal{O}_{X_{1}^{\prime}}$. Then $\mathcal{I O}_{U}$ is locally principal by (30). By axiom 4 and (i), there exists a birational projective morphism $\epsilon: Y_{1} \rightarrow X_{1}^{\prime}$ such that $\mathcal{I} \mathcal{O}_{\epsilon^{-1}\left(U_{2}\right)}$ is locally principal and

$$
\epsilon^{-1}\left(U_{2}\right) \subseteq \operatorname{RegP}\left(Y_{1}\right) \text { and } \epsilon^{-1}(U) \simeq U
$$

By (i) of axiom 2, it can be furthermore assumed that $Y_{1}$ is normal. Let $V_{2}:=$ $\epsilon^{-1}\left(U_{2}\right)$ and $V_{1}:=\eta^{\prime-1}\left(U_{1}\right)$. By $(30)$ and $(31), V_{1}$ and $V_{2}$ glue along $\epsilon^{-1}(U) \simeq \eta^{\prime-1}(U)$ to a proper model $Y / k$ of $K$. On the other hand, $Y$ is projective since $X_{1}^{\prime}$ is projective and both $\epsilon$ and $\eta^{\prime}$ are projective morphisms.

We claim that $Y$ satisfies the conclusion of the proposition. Since $X_{2}^{\prime}$ and $Y_{1}$ are normal, $Y$ is also normal. The rational map $Y \cdots \rightarrow X_{2}^{\prime}$ is defined and is an isomorphism on $V_{1}$; by the universal property of blowing up, $Y \cdots \rightarrow X_{2}^{\prime}$ is defined on 
$V_{2}$; therefore there exists a projective morphism $Y \rightarrow X_{2}^{\prime}$, hence projective morphisms $\pi_{i}: Y \rightarrow X_{i}, i=1,2$. By (i) and (31), we have $\pi_{1}^{-1}\left(\operatorname{RegP}\left(X_{1}\right)\right) \subseteq V_{2} \subseteq \operatorname{RegP}(Y)$; $\pi_{2}^{-1}\left(\operatorname{RegP}\left(X_{2}\right)\right) \cap V_{1} \simeq f_{2}^{-1}\left(\operatorname{RegP}\left(X_{2}\right)\right) \cap V_{1}$, so by $(\mathrm{i}) \pi_{2}^{-1}\left(\operatorname{RegP}\left(X_{2}\right)\right) \cap V_{1} \subseteq \operatorname{RegP}(Y)$ and this concludes the proof of proposition 5.1.

Corollary 5.7. Let $X / k$ be a given projective model of $K$. There exists a normal and projective model $Y / k$ of $K$, together with a morphism $\pi: Y \rightarrow X$ such that $Y=\operatorname{RegP}(Y)$.

Proof. By axiom 5 , for each $k$-valuation ring $V / k$ of $K$, there exists a projective model $X_{V} / k$ of $K$ such that the center $x_{V} \in X_{V}$ of $V$ satisfies $x_{V} \in \operatorname{RegP}\left(X_{V}\right)$. It can be assumed that $X_{V} \cdots \rightarrow X$ is everywhere defined by proposition 3.3. By axiom 1 and quasi-compactness of the space of $k$-valuations of $K$, there exists a finite number of projective models $X_{1}, \ldots, X_{n}$ of $K$ such that the following holds: for any $k$-valuation ring $V / k$ of $K$, there exists $i, 1 \leq i \leq n$ such that the center $x_{i, V}$ of $V$ in $X_{i}$ belongs to $\operatorname{RegP}\left(X_{i}\right)$. By applying $n-1$ consecutive times proposition 5.1, we construct a normal and projective model $Y$ of $K$ such that $Y=\operatorname{RegP}(Y)$ and $Y \rightarrow X$ is a morphism.

6. Eliminating the Fundamental locus: Proof OF theOrem 2.5 .

In this section, $P$ is a regularity property of local rings $R \subseteq K$, essentially of finite type over $k$ and such that $Q F(R)=K$, verifying axioms 1 to 6 . The proof of theorem 2.5 is merely an adaptation of V. Cossart's refinement of Zariski's Patching Theorem ([11] or proposition 4.8 in [12]).

Let $X / k$ be a given normal and projective model of $K$. By corollary 5.7, there exists a normal and projective model $Y_{0}$ of $K$, together with a morphism $\pi_{0}: Y_{0} \rightarrow X$ such that $Y_{0}=\operatorname{RegP}\left(Y_{0}\right)$.

Since $X$ is normal, the fundamental locus $\mathcal{F}_{\pi_{0}}$ of $\pi_{0}^{-1}$ has dimension at most one; we have $\operatorname{SingP}(X) \subseteq \mathcal{F}_{\pi_{0}}$. Let $\mathcal{G}_{0}$ be the union of all irreducible components of $\mathcal{F}_{\pi_{0}}$ which are not identically contained in $\operatorname{SingP}(X)$, so $\mathcal{F}_{\pi_{0}}=\mathcal{G}_{0} \cup \operatorname{SingP}(X)$. Let $Z_{1}, \ldots, Z_{m}$ be those one-dimensional irreducible components of $\mathcal{G}_{0}$ such that $Z_{i} \cap \operatorname{SingP}(X) \neq \emptyset$. Let $g_{1}: X_{1} \rightarrow X$ be the composition of all those morphisms $X^{\prime} \rightarrow X$ given by axiom 6 when applied consecutively to each of $Z_{1}, \ldots, Z_{m}$. It can be assumed w.l.o.g. that the fundamental locus of $g_{1}^{-1}$ is contained in $\operatorname{Sing} \mathrm{P}(X)$ and in particular we have

$$
g_{1}^{-1}(\operatorname{RegP}(X)) \simeq \operatorname{RegP}(X) .
$$

Let $z \in \mathcal{F}_{\pi_{0}}$ be a closed point whose normalized blowing up has been performed in the process of building up $g_{1}$, so $\pi_{0}(z) \in \operatorname{SingP}(X)$, and let $M_{z} \subset \mathcal{O}_{X}$ be the ideal of $z$. By axiom 4 applied to the pair $\left(X, M_{z}\right)$, there exists a projective birational morphism $Y_{1} \rightarrow Y_{0}$ such that $M \mathcal{O}_{Y_{1}}$ is locally principal and $\operatorname{RegP}\left(Y_{1}\right)=Y_{1}$. Note that (iii) of axiom 4 implies that $Y_{1} \rightarrow Y_{0}$ is an isomorphism above $Y_{0} \backslash \pi_{0}^{-1}(\operatorname{SingP}(X))$. By (i) of axiom 2, it can be furthermore assumed that $Y_{1}$ is normal, whence $Y_{1} \rightarrow X$ factors through the normalized blowing up along $z$.

Arguing by induction on the number of normalized blowing up performed in $g_{1}$, we obtain a projective morphism $\eta: X_{2} \rightarrow X_{1}$ such that $\operatorname{RegP}\left(X_{2}\right)=X_{2}$. Let $\Sigma_{0}$ (resp. $Z_{i}^{\prime}$ ) be the strict transform of $\mathcal{G}_{0}\left(\right.$ resp. $\left.Z_{i}, 1 \leq i \leq m\right)$ in $X_{1}$. We have

$$
\Sigma_{0} \subseteq \operatorname{RegP}\left(X_{1}\right) .
$$

By construction, the fundamental locus $\mathcal{F}_{\eta}$ of $\eta^{-1}$ satisfies

$$
\mathcal{F}_{\eta} \subseteq \Sigma_{0} \cup g_{1}^{-1}(\operatorname{SingP}(X)) .
$$


Let $\Sigma_{1}:=\operatorname{SingP}\left(X_{1}\right)$, so by $(33)$ we have

$$
\Sigma_{0} \cap \Sigma_{1}=\emptyset .
$$

The proof is now a variation of steps 3 to 5 of the proof of proposition 5.1. We indicate how to adapt the argument to our case. Recall definition 5.4 of bad points for $\Sigma_{1}$. By lemma 5.3, it can be assumed without loss of generality that $f_{1}$ is factorizable above each $x \in \operatorname{RegP}\left(X_{1}\right), \operatorname{dim} \mathcal{O}_{X_{1}, x}=2$, which is bad for $\Sigma_{1}$. With notations as in (27), we deduce from (33), (34) and (35) that

$$
\Phi \subseteq g_{1}^{-1}(\operatorname{SingP}(X))
$$

with notations as in (27) (note that $\Phi=\Sigma_{1} \cup \Sigma_{b}$ since $\left.\operatorname{RegP}\left(X_{2}\right)=X_{2}\right)$.

By lemma 5.6, (32) and (36), there exists a diagram (29) such that there is no $x^{\prime} \in f_{1}^{-1}\left(\operatorname{RegP}\left(X_{1}\right)\right), \operatorname{dim} \mathcal{O}_{X_{1}^{\prime}, x^{\prime}}=2$, which is bad for $\Sigma_{1}^{\prime}$ and the following holds:

(i) $\operatorname{RegP}\left(X_{2}^{\prime}\right)=X_{2}^{\prime}$;

(ii) $g_{1} \circ f_{1}$ induces an isomorphism $X_{1}^{\prime} \backslash\left(g_{1} \circ f_{1}\right)^{-1}(\operatorname{SingP}(X)) \simeq \operatorname{RegP}(X)$.

Since there is no bad $x^{\prime}$ for $\Sigma_{1}^{\prime}$, any irreducible component of $\mathcal{F}_{\eta^{\prime}}$ is either identically contained in $f_{1}^{-1}\left(\operatorname{RegP}\left(X_{1}\right)\right)$ or is contained in $\Sigma_{1}^{\prime}$. Let $\mathcal{F}_{1}:=\mathcal{F}_{\eta^{\prime}} \cap f_{1}^{-1}\left(\operatorname{RegP}\left(X_{1}\right)\right)$, $U_{1}:=X_{1}^{\prime} \backslash \mathcal{F}_{1}$ and $U_{2}:=f_{1}^{-1}\left(\operatorname{RegP}\left(X_{1}\right)\right) \subseteq \operatorname{RegP}\left(X_{1}^{\prime}\right)$. We have $X_{1}^{\prime}=U_{1} \cup U_{2}$. Let $U:=U_{1} \cap U_{2}$, so by definition of $\mathcal{F}_{\eta^{\prime}}$, we have $\eta^{\prime-1}(U) \simeq U$. Therefore we can glue the open sets $V_{1}:=\eta^{\prime-1}\left(U_{1}\right)$ and $U_{2}$ along $\eta^{-1}(U) \simeq U$ to a proper model $Y / k$ of $K$ such that $\operatorname{RegP}(Y)=Y$ by (i) above. As in step 5 of the proof of proposition 5.1, there is actually a projective morphism $\pi: Y \rightarrow X$. Finally, we have $\pi^{-1}(\operatorname{RegP}(X)) \simeq \operatorname{RegP}(X)$, since $Y \rightarrow X_{1}^{\prime}$ is an isomorphism above $U_{2}$ and $\operatorname{RegP}(X) \simeq\left(g_{1} \circ f_{1}\right)^{-1}(\operatorname{RegP}(X)) \subseteq U_{2}$ by (ii) above.

\section{Open PROBLEMS}

In this section, we summarize the main problem and open questions.

Problem 7.1. Let $P$ be a regularity property verifying axioms 1 to 6 and $X / k$ be a normal projective model of $K$. Under which extra conditions does there exist a sequence of morphisms

$$
X=: X_{0} \leftarrow X_{1} \leftarrow \cdots \leftarrow X_{n}=: X^{\prime},
$$

where $X_{i-1} \leftarrow X_{i}$ is the normalized blowing up along a regular integral subvariety $Z_{i-1} \subseteq \operatorname{SingP}\left(X_{i-1}\right)$ for $1 \leq i \leq n$, such that $\operatorname{RegP}\left(X^{\prime}\right)=X^{\prime}$ ?

If $\operatorname{char} k=0$ and $P=P_{\text {reg }}$ is the usual regularity property, the problem has an affirmative answer for those properties $P_{\mu}, \mu \geq 1$ of example 3.4 , in particular for $P$ itself (with $\mu=1$ ). This is easily deduced from Hironaka's theorem [18] using the construction given in (6) along the proof of proposition 3.5. For as far as we know, the same problem is open if chark $>0$, or in the situation of corollary 4.9 (vector fields), or in that of theorem 4.10 (our weak version of Hironaka's conjecture).

Other open questions have to do with the redundance of our axioms 1 to 6 . If $K / k$ is a function field of dimension two, any regularity property $P$ in $K$ verifying axioms 1, 2 and 5 satisfies the conclusion of (the dimension two version of) theorem 2.5. In 
this case, a variety $X^{\prime}$ as in loc.cit can be constructed by Zariski's algorithm: let $X / k$ be a normal projective model of $K$; any sequence of morphisms

$$
X=: X_{0} \leftarrow X_{1} \leftarrow \cdots \leftarrow X_{n} \cdots,
$$

where $X_{i-1} \leftarrow X_{i}$ is the normalized blowing up of a closed point $z_{i-1} \subseteq \operatorname{SingP}\left(X_{i-1}\right)$ for $1 \leq i \leq n$, is finite. This fact is a simple consequence of Zariski's theorem p.688 [30] on resolution of surface singularities by successive normalized blowing ups along the singular locus and of Abhyankar's theorem 2 [2]. This suggests the following problem:

Problem 7.2. Let $K / k$ be a function field of dimension three. Construct a regularity property $P$ in $K$ verifying axioms 1, 2 and 5 and satisfying (i) below (resp. (ii); (iii)).

(i) $P$ does not verify axiom 3.

(ii) $P$ does not verify axiom 6 .

(iii) $P$ verifies axiom 3, but does not verify axiom 4.

\section{REFERENCES}

[1] Abhyankar, S., Local uniformization on algebraic surfaces over ground fields of characteristic $p \neq 0$, Ann. Math. 63 (1956), 491-526.

[2] Abhyankar, S., On the valuations centered in a local domain, Amer. J. Math. 78 (1956), 321-348.

[3] Abhyankar, S., Resolution of singularities of embedded algebraic surfaces, second edition, Springer Monographs in Math. (1998), Springer Verlag.

[4] Abramovich, D. And De Jong, A.J., Smoothness, semistablility, and toroidal geometry, J. Alg. Geom. 6 (1997), 789-801.

[5] Abramovich, D., Karu, K., Matsuki, K., WŁodarczyk, J., Torification and factorization of birational maps. J. Amer. Math. Soc. 15 (2002), no. 3, 531-572 .

[6] Bogomolov, F., Pantev, T., Weak Hironaka Theorem, Math. Res. Lett. 3 (1996), 299-307.

[7] CAno, F., Desingularization strategies for three-dimensional vector fields, Lecture Notes in Mathematics 1259 (1987), Springer-Verlag, Berlin.

[8] Cano, F., Reduction of the singularities of nondicritical singular foliations. Dimension three. Amer. J. Math. 115 (1993), no. 3, 509-588.

[9] Cano, F., Reduction of the singularities of codimension one singular foliations in dimension three, Ann. of Math. 160 (2004), no. 3, 907-1011.

[10] Cano, F., Roche, C., Spivakovsky, M., Reduction of singularities of three-dimensional line foliations, this volume.

[11] Cossart, V., Modèle projectif régulier et désingularisation, Math. Ann. 293(1) (1992), 115-122.

[12] Cossart, V. And Piltant, O.,Cossart, Resolution of singularities of threefolds in positive characteristic. I. Reduction to local uniformization on Artin-Schreier and purely inseparable coverings, J. Algebra 320 (2008), no. 3, 1051-1082.

[13] Cossart, V. and Piltant, O., Resolution of singularities of threefolds in positive characteristics II, J. Algebra 321 (2009), no. 1, 1836-1976.

[14] Cutkosky, S.D., Local monomialization and factorization of morphisms, Astérisque 260 (1999).

[15] Cutkosky, S.D., Toroidalization of dominant morphisms of 3-folds, Mem. Amer. Math. Soc. 190 (2007).

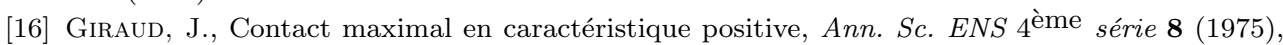
201-234.

[17] Hartshorne, R., Algebraic Geometry, Graduate Texts in Mathematics 52 (1977), SpringerVerlag.

[18] Hironaka, H., Resolution of singularities of an algebraic variety over a field of characteristic zero, Ann. Math. 79 (1964), 109-326.

[19] Hironaka, H., Desingularization of excellent surfaces, Advanced Science Seminar in Algebraic Geometry (1967), Bowdoin College, Brunswick, Maine, 1967.

[20] Hironaka, H., Additive groups associated with points of a projective space, Ann. Math. 92 (1970), 327-334.

[21] HironakA, H., Idealistic exponents of singularity, in J.J. Sylvester symposium, John Hopkins Univ. (Baltimore 1976), John Hopkins Univ. Press (1977), 52-125. 
[22] De Jong, A.J., Smoothness, semistability and Alterations, Publ. Math. I.H.E.S. 83 (1996), 51-93.

[23] Karu, K., Local strong factorization of toric birational maps, J. Algebraic Geom. 14 (2005), 165-175.

[24] Lipman, J., Introduction to resolution of singularities, in Algebraic Geometry, Arcata 1974, Amer. Math. Soc. Proc. Symp. Pure Math. 29 (1975), 187-230.

[25] Lipman, J., Desingularization of two-dimensional schemes, Ann. Math. 107 (1978), 151-207.

[26] Matsumura, H., Commutative ring theory, Cambridge studies in advanced mathematics 8 (1986), Cambridge Univ. Press.

[27] Pinkham, H., Factorization of birational maps in dimension three, Proc. Symp. in Pure Math., 40 Part 2, AMS Providence (1983), 343-372.

[28] Seidenberg, A., Reduction of singularities of the differential equation $A d y=B d x$, Amer. $J$. Math 90 (1968), 248-269.

[29] WŁodarczyk, J., Toroidal varieties and the weak factorization theorem, Invent. Math. 154 (2003), no. 2, 223-331.

[30] ZARISKI, O., The reduction of the singularities of an algebraic surface, Ann. Math. 40 (1939), 639-689.

[31] ZARISKi, O., Local uniformization of algebraic varieties, Ann. Math. 41 (1940), 852-896.

[32] ZARISKI, O., The compactness of the Riemann manifold of an abstract field of algebraic functions, Bull. Amer. Math. Soc. 45 (1944), 683-691.

[33] ZARISKI, O., Reduction of the singularities of algebraic three dimensional varieties, Ann. Math. 45 (1944), 472-542.

[34] Zariski, O. And Samuel, P., Commutative Algebra II (1960), Van Nostrand, Princeton. 